\title{
A Theory of Truncated Inverse Sampling *
}

\author{
Xinjia Chen
}

November 2008

\begin{abstract}
In this paper, we have established a new framework of truncated inverse sampling for estimating mean values of non-negative random variables such as binomial, Poisson, hypergeometrical, and bounded variables. We have derived explicit formulas and computational methods for designing sampling schemes to ensure prescribed levels of precision and confidence for point estimators. Moreover, we have developed interval estimation methods.
\end{abstract}

\section{Introduction}

Parametric estimation based on sampling is an important branch of mathematical statistics with ubiquitous applications across many fields, from operation research, biology and medical science, agriculture science, computer science, social science, telecommunication engineering, control engineering, to name a few. A wide class estimation problems of both theoretical and practical significance can be put into the setting of estimating the mean value of a random variable via sampling. Familiar examples include the estimation of binomial parameters, Poisson parameters, finite population proportion, the mean of a bounded variable, and so on. A simple yet frequently used sampling scheme for estimating the mean value a random variable $X$ is to draw samples of $X$ until the sample sum is no less than a prescribed threshold and then take the empirical mean as an estimate for the true mean value. This sampling scheme, referred to as inverse sampling, was first studied by Haldane [8, 9] in the context of estimating a binomial parameter. Recently, inverse sampling has been studied by Chen [1, 2, Dagum et al. [5] and Cheng [3] for estimation of the mean of a bounded variable. Mendo and Hernando [10] have revisited inverse sampling for estimating binomial parameters.

Theoretically, there is no limit on the number of samples for inverse sampling. However, the practical situation is quite contrary. Due to the limitation of resources, almost every practitioner would specify a maximum sample size on the sampling. This means that the frequently used

\footnotetext{
${ }^{*}$ The author had been previously working with Louisiana State University at Baton Rouge, LA 70803, USA, and is now with Department of Electrical Engineering, Southern University and A\&M College, Baton Rouge, LA 70813, USA; Email: chenxinjia@gmail.com
} 
method is actually the truncated inverse sampling scheme in the sense that sampling is continued until the sample sum is no less than a prescribed threshold or the number of samples reach the maximum sample size.

While the ideal inverse sampling has drawn extensive research effort, little attention has been paid to the theoretical issues of the truly useful truncated inverse sampling scheme. In this paper, we shall investigate the essential theory of truncated inverse sampling with a prevailing theme of error control. We have answered two equally central problems regarding pre-experimental planning and post-experimental analysis. The first problem is on the determination of the threshold value and the maximum sample size for guaranteeing prescribed levels of precision and confidence of an estimator. The second problem is on interval estimation of the parameter based on the observed data when the truncated inverse sampling is completed.

The remainder of the paper is organized as follows. In Section 2, we present our general results for truncated inverse sampling. In Section 3, we consider the problem of estimating binomial parameters. In Section 4, we discuss the estimation of the proportion of a finite proportion. In Section 5, we discuss the estimation of Poisson parameters. The estimation of the mean of a bounded variable is investigated in Section 6. Section 7 is the conclusion.

Throughout this paper, we shall use the following notations. The expectation of a random variable is denoted by $\mathbb{E}[$.$] . The set of positive integers is denoted by \mathbb{N}$. The ceiling function and floor function are denoted respectively by $\lceil$.$\rceil and \lfloor$.$\rfloor (i.e., \lceil x\rceil$ represents the smallest integer no less than $x ;\lfloor x\rfloor$ represents the largest integer no greater than $x$ ). The gamma function is denoted by $\Gamma($.$) . For any integer m$, the combinatoric function $\left(\begin{array}{c}m \\ z\end{array}\right)$ with respect to integer $z$ takes value

$\frac{\Gamma(m+1)}{\Gamma(z+1) \Gamma(m-z+1)}$ for $z \leq m$ and value 0 otherwise. The left limit as $\epsilon$ tends to 0 is denoted as $\lim _{\epsilon \downarrow 0}$.

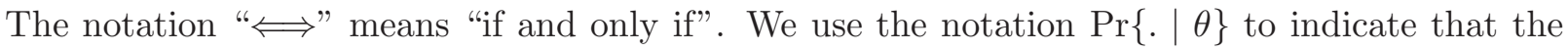
associated random samples $X_{1}, X_{2}, \cdots$ are parameterized by $\theta$. The parameter $\theta$ in $\operatorname{Pr}\{. \mid \theta\}$ may be dropped whenever this can be done without introducing confusion. The other notations will be made clear as we proceed.

\section{General Theory}

In this section, we shall develop some general results on the truncated inverse sampling. Let $X$ be a non-negative random variable defined in a probability space $(\Omega, \mathscr{F}, \operatorname{Pr})$. Our problem is to estimate the mean, $\mu=\mathbb{E}[X]$, of $X$ based on i.i.d. random samples $X_{1}, X_{2}, \cdots$ of $X$. To this end, we shall adopt a truncated inverse sampling scheme as follows:

Continue sampling until the sample sum is no less than a threshold value $\gamma>0$ or the number of samples reaches an integer $n$.

Let $\mathbf{n}$ be the total number of samples when the sampling is stopped. By the definition of the truncated inverse sampling scheme, $\mathbf{n}$ is a random variable such that

$$
\mathbf{n}(\omega)=\min \left\{n, \min \left\{\ell \in \mathbb{N}: \sum_{i=1}^{\ell} X_{i}(\omega) \geq \gamma\right\}\right\}
$$


for any $\omega \in \Omega$. Define $\mathbf{k}=\sum_{i=1}^{\mathbf{n}} X_{i}$. Then, we can take $\widehat{\boldsymbol{\mu}}=\frac{\min \{\mathbf{k}, \gamma\}}{\mathbf{n}}$ as the estimator for $\mu=\mathbb{E}[X]$.

With regard to the distribution of $\widehat{\boldsymbol{\mu}}$, we have

Theorem 1 For any $z>0$,

$$
\operatorname{Pr}\{\widehat{\boldsymbol{\mu}} \leq z\}=\left\{\begin{array}{ll}
\operatorname{Pr}\left\{\sum_{i=1}^{\lceil\gamma / z\rceil-1} X_{i}<\gamma\right\} & \text { for } \gamma \leq n z, \\
\operatorname{Pr}\left\{\sum_{i=1}^{n} X_{i} \leq n z\right\} & \text { for } \gamma>n z
\end{array} \quad \operatorname{Pr}\{\widehat{\boldsymbol{\mu}} \geq z\}= \begin{cases}\operatorname{Pr}\left\{\sum_{i=1}^{\lfloor\gamma / z\rfloor} X_{i} \geq \gamma\right\} & \text { for } \gamma \leq n z, \\
\operatorname{Pr}\left\{\sum_{i=1}^{n} X_{i} \geq n z\right\} & \text { for } \gamma>n z .\end{cases}\right.
$$

With regard to the average sample number $\mathbb{E}[\mathbf{n}]$ of the truncated inverse sampling associated with random variable $X$, we have

Theorem 2 For any non-negative random variable $X$ with positive mean and finite variance, $\mathbb{E}[\mathbf{n}]<\min \left\{n, \frac{\gamma}{\mu}+1\right\}$. Specially, if $\gamma$ is a positive integer and $X$ is a Bernoulli random variable such that $\operatorname{Pr}\{X=1\}=1-\operatorname{Pr}\{X=0\}=p \in(0,1)$, then $\mathbb{E}[\mathbf{n}]<\min \left\{n, \frac{\gamma}{p}\right\}$.

\section{Estimation of Binomial Parameters}

In this section, we shall consider the estimation of a binomial parameter based on truncated inverse sampling. Let $X$ be a Bernoulli random variable such that $\operatorname{Pr}\{X=1\}=1-\operatorname{Pr}\{X=$ $0\}=p \in(0,1)$. Our goal is to estimate $p$ based on i.i.d. random samples $X_{1}, X_{2}, \cdots$ of $X$. Since $X_{i}$ assumes only two possible values 0 or 1 , the threshold value $\gamma$ shall be restricted to an integer. The estimator for $p$ can be taken as $\widehat{\boldsymbol{p}}=\frac{\min \{\mathbf{k}, \gamma\}}{\mathbf{n}}=\frac{\mathbf{k}}{\mathbf{n}}$, where $\mathbf{k}$ and $\mathbf{n}$ have been defined in Section 2 .

In order to estimate $p$ via truncated inverse sampling, a critical problem is the determination of the threshold value $\gamma$ and the maximum sample size $n$. By making use of functions

$$
\mathscr{M}_{\mathrm{B}}(z, \mu)=z \ln \left(\frac{\mu}{z}\right)+(1-z) \ln \left(\frac{1-\mu}{1-z}\right), \quad \mathscr{M}_{\mathrm{I}}(z, \mu)=\frac{1}{z} \mathscr{M}_{\mathrm{B}}(z, \mu)
$$

for $0<z<1$ and $0<\mu<1$, we have derived the following result.

Theorem 3 Let $0<\delta<1$. Let $0<\varepsilon_{a}<\varepsilon_{r}<1$ be respectively the margins of absolute and relative errors such that $\frac{\varepsilon_{a}}{\varepsilon_{r}}+\varepsilon_{a} \leq \frac{1}{2}$. Then, $\operatorname{Pr}\left\{|\widehat{\boldsymbol{p}}-p|<\varepsilon_{a}\right.$ or $\left.\left|\frac{\widehat{\boldsymbol{p}}-p}{p}\right|<\varepsilon_{r}\right\}>1-\delta$ provided that $n>\frac{\ln (\delta / 2)}{\mathscr{M}_{\mathrm{B}}\left(p^{\star}+\varepsilon_{a}, p^{\star}\right)}$ and $\gamma>\frac{\ln (\delta / 2)}{\mathscr{M}_{\mathrm{I}}\left(p^{\star}+\varepsilon_{a}, p^{\star}\right)}$ where $p^{\star}=\frac{\varepsilon_{a}}{\varepsilon_{r}}$.

Theorem 3 provides explicit formulas for determining the threshold value $\gamma$ and the maximum sample size $n$. To reduce conservatism, we can take a computational approach to obtain smaller $\gamma$ and $n$. In this direction, the following theorem which is of fundamental importance.

Theorem 4 Let $\mathscr{L}($.$) and \mathscr{U}($.$) be monotone functions. Let the supports of \mathscr{L}(\widehat{\boldsymbol{p}})$ and $\mathscr{U}(\widehat{\boldsymbol{p}})$ be denoted by $I_{\mathscr{L}}$ and $I_{\mathscr{U}}$ respectively. Then, the maximum of $\operatorname{Pr}\{p \leq \mathscr{L}(\widehat{\boldsymbol{p}}) \mid p\}$ with respect to $p \in[a, b] \subseteq[0,1]$ is achieved at $I_{\mathscr{L}} \cap[a, b] \cup\{a, b\}$ provided that $I_{\mathscr{L}}$ has no closure point in $[a, b]$. Similarly, the maximum of $\operatorname{Pr}\{p \geq \mathscr{U}(\widehat{\boldsymbol{p}}) \mid p\}$ with respect to $p \in[a, b] \subseteq[0,1]$ is achieved at $I_{\mathscr{U}} \cap[a, b] \cup\{a, b\}$ provided that $I_{\mathscr{U}}$ has no closure point in $[a, b]$. 
In Theorem 4, we have used the concept of support. The support of a random variable is referred to the set of all possible values that the random variable can assume. By virtue of Theorem 4, we have obtained the following results.

Theorem 5 Let $0<\delta<1$ and $\zeta>0$. Let $0<\varepsilon_{a}<\varepsilon_{r}<1$ be respectively the margins of absolute and relative errors. Define $n=\left\lfloor\frac{\ln (\zeta \delta)}{\mathscr{M}_{\mathrm{B}}\left(p^{\star}+\varepsilon_{a}, p^{\star}\right)}\right\rfloor$ and $\gamma=\left\lfloor\frac{\ln (\zeta \delta)}{\mathscr{M}_{\mathrm{I}}\left(p^{\star}+\varepsilon_{a}, p^{\star}\right)}\right\rfloor$ with $p^{\star}=\frac{\varepsilon_{a}}{\varepsilon_{r}}$. Define $\mathscr{Q}_{a}^{-}$as the support of $\widehat{\boldsymbol{p}}-\varepsilon_{a}, \mathscr{Q}_{a}^{+}$as the support of $\widehat{\boldsymbol{p}}+\varepsilon_{a}, \mathscr{Q}_{r}^{+}$as the support of $\widehat{\boldsymbol{p}} /\left(1+\varepsilon_{r}\right)$, $\mathscr{Q}_{r}^{-}$as the support of $\widehat{\boldsymbol{p}} /\left(1-\varepsilon_{r}\right)$. Then, $\operatorname{Pr}\left\{|\widehat{\boldsymbol{p}}-p|<\varepsilon_{a}\right.$ or $\left.\left|\frac{\widehat{\boldsymbol{p}}-p}{p}\right|<\varepsilon_{r}\right\}>1-\delta$ provided that

$$
\begin{aligned}
& \operatorname{Pr}\left\{\widehat{\boldsymbol{p}} \geq p+\varepsilon_{a} \mid p\right\} \leq \frac{\delta}{2}, \quad \forall p \in \mathscr{Q}_{a}^{-} \cup\left\{p^{\star}\right\} \cap\left(0, p^{\star}\right] \\
& \operatorname{Pr}\left\{\widehat{\boldsymbol{p}} \leq p-\varepsilon_{a} \mid p\right\} \leq \frac{\delta}{2}, \quad \forall p \in \mathscr{Q}_{a}^{+} \cup\left\{p^{\star}\right\} \cap\left(0, p^{\star}\right] \\
& \operatorname{Pr}\left\{\widehat{\boldsymbol{p}} \geq p\left(1+\varepsilon_{r}\right) \mid p\right\} \leq \frac{\delta}{2}, \\
& \operatorname{Pr}\left\{\widehat{\boldsymbol{p}} \leq p\left(1-\varepsilon_{r}\right) \mid p\right\} \leq \frac{\delta}{2}, \quad \forall p \in \mathscr{Q}_{r}^{+} \cap\left(p^{\star}, 1\right)
\end{aligned}
$$

where these conditions are satisfied when $\zeta$ is smaller than $\frac{1}{2}$.

Clearly, the support of $\widehat{\boldsymbol{p}}$ is $\left\{\frac{j}{n}: j=0,1, \cdots, \gamma\right\} \cup\left\{\frac{\gamma}{m}: m=\gamma, \gamma+1, \cdots, n-1\right\}$. Theorem 5 asserts that the prescribed levels of precision and confidence can be guaranteed if $\zeta$ is small enough. Hence, we can determine an appropriate value of $\zeta$ by a bisection search method.

When the sampling is terminated, it is desirable to construct a confidence interval for $p$. For this purpose, we have

Theorem 6 Let $0<\delta<1$. Define lower confidence limit $\underline{\boldsymbol{p}} \in[0,1)$ such that $\underline{\boldsymbol{p}}=0$ for $\mathbf{k}=0$ and that $\sum_{i=\mathbf{k}}^{\mathbf{n}}\left(\begin{array}{c}\mathbf{n} \\ i\end{array}\right) \underline{\boldsymbol{p}}^{i}(1-\underline{\boldsymbol{p}})^{\mathbf{n}-i}=\frac{\delta}{2}$ for $\mathbf{k}>0$. Define upper confidence limit $\overline{\boldsymbol{p}} \in(0,1]$ such that $\overline{\boldsymbol{p}}=1$ for $\mathbf{k}=\mathbf{n}$ and that $\sum_{i=0}^{\mathbf{k}}\left(\begin{array}{c}\mathbf{n} \\ i\end{array}\right) \overline{\boldsymbol{p}}^{i}(1-\overline{\boldsymbol{p}})^{\mathbf{n}-i}=\frac{\delta}{2}$ for $\mathbf{k}<\mathbf{n}$. Then, $\operatorname{Pr}\{\underline{\boldsymbol{p}}<p<\overline{\boldsymbol{p}}\} \geq 1-\delta$.

It should be noted the approach of constructing a confidence interval for $p$ can be considered as a generalization of Clopper and Pearson's method [4] of interval estimation.

\section{Estimation of Finite Population Proportion}

In the last section, we have investigated the estimation of a binomial parameter $p$, which can be considered as the proportion of an infinite population. In many situations, the population size is finite and we shall devote this section to the estimation of the proportion of a finite population. Consider a population of $N$ units, among which there are $M$ units having a certain attribute. It is a

frequent problem to estimate the population proportion $p=\frac{M}{N}$ by sampling without replacement. The procedure of sampling without replacement can be precisely described as follows: 
Each time a single unit is drawn without replacement from the remaining population so that every unit of the remaining population has equal chance of being selected.

Such a sampling process can be exactly characterized by random variables $X_{1}, \cdots, X_{N}$ defined in a probability space $(\Omega, \mathscr{F}, \operatorname{Pr})$ such that $X_{i}$ denotes the characteristics of the $i$-th sample in the sense that $X_{i}=1$ if the $i$-th sample has the attribute and $X_{i}=0$ otherwise. By the nature of the sampling procedure, it can be shown that

$$
\operatorname{Pr}\left\{X_{i}=x_{i}, i=1, \cdots, n\right\}=\left(\begin{array}{c}
M \\
\sum_{i=1}^{n} x_{i}
\end{array}\right)\left(\begin{array}{c}
N-M \\
n-\sum_{i=1}^{n} x_{i}
\end{array}\right) /\left[\left(\begin{array}{c}
n \\
\sum_{i=1}^{n} x_{i}
\end{array}\right)\left(\begin{array}{l}
N \\
n
\end{array}\right)\right]
$$

for any $n \in\{1, \cdots, N\}$ and any $x_{i} \in\{0,1\}, i=1, \cdots, n$. Moreover, if the proportion $p=\frac{M}{N}$ is fixed and the population size $N$ tends to infinity, the sequence $X_{1}, X_{2}, \cdots, X_{N}$ tends to the i.i.d. random samples of a Bernoulli variable.

To estimate the population proportion $p$, we can use a sampling scheme defined by positive integers $\gamma$ and $n$ as follows:

Continue sampling without replacement until $\gamma$ units found to have a certain attribute or the number of samples reaches $n$.

Despite the lack of independence in the sequence $X_{1}, X_{2}, \cdots, X_{N}$ with joint distribution (55), such a sampling method is also referred to as truncated inverse sampling due to the fact that, when the sampling is terminated, the number of units having a certain attribute, denoted by $\mathbf{k}$, is actually equal to $\sum_{i=1}^{\mathbf{n}} X_{i}$, where $\mathbf{n}$ is the sample size when the sampling is terminated. This implies that, by relaxing the independency assumption, we can put such a sampling scheme in the general framework of truncated inverse sampling described in Section 2. It can be seen that, as the sample size tends to infinity while the proportion $p$ is being fixed, such a sampling scheme reduces to the truncated inverse sampling for the estimation of a binomial parameter as discussed in Section 3.

As in the case of estimating a binomial parameter in Section 3, the estimator for the proportion of a finite population can be taken as $\widehat{\boldsymbol{p}}=\frac{\min \{\mathbf{k}, \gamma\}}{\mathbf{n}}=\frac{\mathbf{k}}{\mathbf{n}}$. In order to determine $n$ and $\gamma$ to guarantee prescribed levels of precision and confidence, we have the following result.

Theorem 7 Let $0<\delta<1$. Let $0<\varepsilon_{a}<\varepsilon_{r}<1$ be respectively the margins of absolute and relative errors such that $\frac{\varepsilon_{a}}{\varepsilon_{r}}+\varepsilon_{a} \leq \frac{1}{2}$. Then, $\operatorname{Pr}\left\{|\widehat{\boldsymbol{p}}-p|<\varepsilon_{a}\right.$ or $\left.|\widehat{\boldsymbol{p}}-p|<p \varepsilon_{r}\right\}>1-\delta$ provided that $n>\frac{\ln (\delta / 2)}{\mathscr{M}_{\mathrm{B}}\left(p^{\star}+\varepsilon_{a}, p^{\star}\right)}$ and $\gamma>\frac{\ln (\delta / 2)}{\mathscr{M}_{\mathrm{I}}\left(p^{\star}+\varepsilon_{a}, p^{\star}\right)}$, where $p^{\star}=\frac{\varepsilon_{a}}{\varepsilon_{r}}$.

Theorem 7 provides explicit formulas for determining threshold value $\gamma$ and the maximum sample size $n$. To reduce conservatism, we can take a computational approach to obtain smaller $\gamma$ and $n$. In this direction, the following theorem is useful.

Theorem 8 Let $\mathscr{L}($.$) and \mathscr{U}($.$) be non-decreasing integer-valued functions. Let the supports$ of $\mathscr{L}(\widehat{\boldsymbol{p}})$ and $\mathscr{U}(\widehat{\boldsymbol{p}})$ be denoted by $I_{\mathscr{L}}$ and $I_{\mathscr{U}}$ respectively. Then, the maximum of $\operatorname{Pr}\{M \leq$ $\mathscr{L}(\widehat{\boldsymbol{p}}) \mid M\}$ with respect to $M \in[a, b] \subseteq[0, N]$, where $a$ and $b$ are integers, is achieved at 
$I_{\mathscr{L}} \cap[a, b] \cup\{a, b\}$. Similarly, the maximum of $\operatorname{Pr}\{M \geq \mathscr{U}(\widehat{\boldsymbol{p}}) \mid M\}$ with respect to $M \in[a, b]$ is achieved at $I_{\mathscr{U}} \cap[a, b] \cup\{a, b\}$.

By virtue of Theorem 8 , we have obtained the following results.

Theorem 9 Let $0<\delta<1$ and $\zeta>0$. Let $0<\varepsilon_{a}<\varepsilon_{r}<1$ be respectively the margins of absolute and relative errors. Define $n=\left\lfloor\frac{\ln (\zeta \delta)}{\mathscr{M}_{\mathrm{B}}\left(p^{\star}+\varepsilon_{a}, p^{\star}\right)}\right\rfloor$ and $\gamma=\left\lfloor\frac{\ln (\zeta \delta)}{\mathscr{M}_{\mathrm{I}}\left(p^{\star}+\varepsilon_{a}, p^{\star}\right)}\right\rfloor$ with $p^{\star}=\frac{\varepsilon_{a}}{\varepsilon_{r}}$. Define $\mathscr{Q}_{a}^{-}$as the support of $\left\lfloor N\left(\widehat{\boldsymbol{p}}-\varepsilon_{a}\right)\right\rfloor, \mathscr{Q}_{a}^{+}$as the support of $\left\lceil N\left(\widehat{\boldsymbol{p}}+\varepsilon_{a}\right)\right\rceil, \mathscr{Q}_{r}^{+}$as the support of $\left\lfloor N \widehat{\boldsymbol{p}} /\left(1+\varepsilon_{r}\right)\right\rfloor$, $\mathscr{Q}_{r}^{-}$as the support of $\left\lceil N \widehat{\boldsymbol{p}} /\left(1-\varepsilon_{r}\right)\right\rceil$. Then, $\operatorname{Pr}\left\{|\widehat{\boldsymbol{p}}-p|<\varepsilon_{a}\right.$ or $\left.|\widehat{\boldsymbol{p}}-p|<p \varepsilon_{r}\right\}>1-\delta$ provided that

$$
\begin{array}{ll}
\operatorname{Pr}\left\{\widehat{\boldsymbol{p}} \geq p+\varepsilon_{a} \mid M\right\} \leq \frac{\delta}{2}, & \forall M \in \mathscr{Q}_{a}^{-} \cup\left\{\left\lfloor N p^{\star}\right\rfloor\right\} \cap\left(0, N p^{\star}\right] \\
\operatorname{Pr}\left\{\widehat{\boldsymbol{p}} \leq p-\varepsilon_{a} \mid M\right\} \leq \frac{\delta}{2}, & \forall M \in \mathscr{Q}_{a}^{+} \cup\left\{\left\lfloor N p^{\star}\right\rfloor\right\} \cap\left(0, N p^{\star}\right] \\
\operatorname{Pr}\left\{\widehat{\boldsymbol{p}} \geq p\left(1+\varepsilon_{r}\right) \mid M\right\} \leq \frac{\delta}{2}, & \forall M \in \mathscr{Q}_{r}^{+} \cup\left\{\left\lfloor N p^{\star}\right\rfloor+1\right\} \cap\left(N p^{\star}, N\right) \\
\operatorname{Pr}\left\{\widehat{\boldsymbol{p}} \leq p\left(1-\varepsilon_{r}\right) \mid M\right\} \leq \frac{\delta}{2}, & \forall M \in \mathscr{Q}_{r}^{-} \cup\left\{\left\lfloor N p^{\star}\right\rfloor+1\right\} \cap\left(N p^{\star}, N\right)
\end{array}
$$

where these conditions are satisfied when $\zeta$ is smaller than $\frac{1}{2}$.

Clearly, the support of $\widehat{\boldsymbol{p}}$ is $\left\{\frac{j}{n}: j=0,1, \cdots, \gamma\right\} \cup\left\{\frac{\gamma}{m}: m=\gamma, \gamma+1, \cdots, n-1\right\}$. It is asserted by Theorem 9 that the prescribed levels of precision and confidence can be guaranteed if $\zeta$ is small enough. Therefore, an appropriate value of $\zeta$ can be determined by a bisection search method.

In order to construct a confidence interval for $M$, we have

Theorem 10 Let $\boldsymbol{M}_{l}$ be the smallest integer such that $\sum_{i=\mathbf{k}}^{\mathbf{n}}\left(\begin{array}{c}\boldsymbol{M}_{i} \\ i\end{array}\right)\left(\begin{array}{c}N-\boldsymbol{M}_{l} \\ \mathbf{n}-i\end{array}\right) /\left(\begin{array}{c}N \\ \mathbf{n}\end{array}\right)>\frac{\delta}{2}$. Let $\boldsymbol{M}_{u}$ be the largest integer such that $\sum_{i=0}^{\mathbf{k}}\left(\begin{array}{c}\boldsymbol{M}_{i} \\ i\end{array}\right)\left(\begin{array}{c}N-\boldsymbol{M}_{u} \\ \mathbf{n}-i\end{array}\right) /\left(\begin{array}{c}N \\ \mathbf{n}\end{array}\right)>\frac{\delta}{2}$. Then, $\operatorname{Pr}\left\{\boldsymbol{M}_{l} \leq M \leq \boldsymbol{M}_{u}\right\} \geq 1-\delta$.

With regard to the average sample number $\mathbb{E}[\mathbf{n}]$, we have

Theorem 11 If the population proportion $p$ is positive, then $\mathbb{E}[\mathbf{n}]<\min \left\{n, \frac{\gamma}{p}\right\}$.

\section{Estimation of Poisson Parameters}

Let $X$ be a Poisson random variable with mean $\lambda>0$. It is a frequent problem to estimate $\lambda$ based on i.i.d. random samples $X_{1}, X_{2}, \cdots$ of $X$. This can be accomplished by using the truncated inverse sampling scheme described in Section 2. Since $X_{i}$ is an integer-valued random variable, we shall restrict the threshold $\gamma$ to be a positive integer. We take $\widehat{\boldsymbol{\lambda}}=\frac{\min \{\mathbf{k}, \gamma\}}{\mathbf{n}}$ as the estimator for $\lambda$, where $\mathbf{k}$ and $\mathbf{n}$ have been defined in Section 2 .

To determine the threshold $\gamma$ and the maximum sample size $n$, we need to have an upper bound for $\lambda$. We do not pursue results along this line. We are more interested in the construction of a confidence interval when the sampling is completed. For this purpose, we have 
Theorem 12 Let $0<\delta<1$. Define lower confidence limit $\underline{\boldsymbol{\lambda}}$ such that $\underline{\boldsymbol{\lambda}}=0$ for $\hat{\boldsymbol{\lambda}}=0$, $\sum_{i=\gamma}^{\infty} \frac{1}{i !}(\mathbf{n} \underline{\boldsymbol{\lambda}})^{i} \exp (-\mathbf{n} \underline{\boldsymbol{\lambda}})=\frac{\delta}{2}$ for $\hat{\boldsymbol{\lambda}} \geq \frac{\gamma}{n}$, and $\sum_{i=\mathbf{k}}^{\infty} \frac{1}{i !}(n \underline{\boldsymbol{\lambda}})^{i} \exp (-n \underline{\boldsymbol{\lambda}})=\frac{\delta}{2}$ for $0<\hat{\boldsymbol{\lambda}}<\frac{\gamma}{n}$, where $\mathbf{k}=\sum_{i=1}^{n} X_{i}$. Define upper confidence limit $\overline{\boldsymbol{\lambda}}$ such that $\overline{\boldsymbol{\lambda}}=\infty$ for $\mathbf{n}=1$, $\sum_{i=0}^{\gamma-1} \frac{1}{i !}[(\mathbf{n}-$ $1) \overline{\boldsymbol{\lambda}}]^{i} \exp (-(\mathbf{n}-1) \overline{\boldsymbol{\lambda}})=\frac{\delta}{2}$ for $\frac{\gamma}{n} \leq \widehat{\boldsymbol{\lambda}}<\gamma$, and $\sum_{i=0}^{\mathrm{k}} \frac{1}{i !}(n \overline{\boldsymbol{\lambda}})^{i} \exp (-n \overline{\boldsymbol{\lambda}})=\frac{\delta}{2}$ for $\hat{\boldsymbol{\lambda}}<\frac{\gamma}{n}$. Then, $\operatorname{Pr}\{\underline{\boldsymbol{\lambda}}<\lambda<\overline{\boldsymbol{\lambda}}\} \geq 1-\delta$.

It should be noted that the interval estimation method described in Theorem 12 is a generalization of Garwood's interval estimation method [6].

\section{Estimation of Bounded-Variable Means}

Let $X$ be a random variable bounded in $[0,1]$ with mean $\mu=\mathbb{E}[X]$. In many situations, it is desirable to estimate $\mu$ based on i.i.d. random samples $X_{1}, X_{2}, \cdots$ of $X$ (see, e.g., [5] and the references therein). To fulfill this goal, we shall make use of the truncated inverse sampling scheme described in Section 2. In order to determine the threshold $\gamma$ and maximum sample size $n$ to guarantee prescribed levels of precision and confidence, we have

Theorem 13 Let $0<\delta<1$. Let $0<\varepsilon_{a}<\varepsilon_{r}<1$ be respectively the margins of absolute and relative errors such that $p^{\star}+\varepsilon_{a} \leq \frac{1}{2}$ with $p^{\star}=\frac{\varepsilon_{a}}{\varepsilon_{r}}$. Then, $\operatorname{Pr}\left\{|\widehat{\boldsymbol{\mu}}-\mu|<\varepsilon_{a}\right.$ or $\left.\left|\frac{\widehat{\boldsymbol{\mu}}-\mu}{\mu}\right|<\varepsilon_{r}\right\}>1-\delta$ provided that

$$
\gamma>\frac{1-\varepsilon_{r}}{\varepsilon_{r}}, \quad \gamma>\frac{\ln \frac{\delta}{2}}{\mathscr{M}_{\mathrm{I}}\left(\frac{\gamma\left(p^{\star}-\varepsilon_{a}\right)}{\gamma-1+\varepsilon_{r}}, p^{\star}\right)}, \quad \gamma>\frac{\ln \frac{\delta}{2}}{\mathscr{M}_{\mathrm{I}}\left(p^{\star}+\varepsilon_{a}, p^{\star}\right)}, \quad n>\frac{\ln \frac{\delta}{2}}{\mathscr{M}_{\mathrm{B}}\left(p^{\star}+\varepsilon_{a}, p^{\star}\right)} .
$$

With regard to the interval estimation of $\mu$, we have

Theorem 14 Let $0<\delta<1$. Define lower confidence limit $\underline{\boldsymbol{\mu}} \in[0, \widehat{\boldsymbol{\mu}}]$ such that $\underline{\boldsymbol{\mu}}=0$ for $\widehat{\boldsymbol{\mu}}=0$, $\mathscr{M}_{\mathrm{B}}(\widehat{\boldsymbol{\mu}}, \underline{\boldsymbol{\mu}})=\frac{\ln \frac{\delta}{2}}{n}$ for $0<\widehat{\boldsymbol{\mu}}<\frac{\gamma}{n}$, and $\mathscr{M}_{\mathrm{I}}(\widehat{\boldsymbol{\mu}}, \underline{\boldsymbol{\mu}})=\frac{\ln \frac{\delta}{2}}{\gamma}$ for $\widehat{\boldsymbol{\mu}} \geq \frac{\gamma}{n}$. Define upper confidence limit $\overline{\boldsymbol{\mu}} \in[\widehat{\boldsymbol{\mu}}, 1]$ and that $\overline{\boldsymbol{\mu}}=1$ for $\widehat{\boldsymbol{\mu}} \geq \frac{\gamma}{\gamma+1}, \mathscr{M}_{\mathrm{B}}(\widehat{\boldsymbol{\mu}}, \overline{\boldsymbol{\mu}})=\frac{\ln \frac{\delta}{2}}{n}$ for $\widehat{\boldsymbol{\mu}}<\frac{\gamma}{n}, \mathscr{M}_{\mathrm{I}}\left(\frac{\widehat{\boldsymbol{\mu}} \gamma}{\gamma-\widehat{\boldsymbol{\mu}}}, \overline{\boldsymbol{\mu}}\right)=\frac{\ln \frac{\delta}{2}}{\gamma}$ and $\frac{\widehat{\boldsymbol{\mu}} \gamma}{\gamma-\hat{\boldsymbol{\mu}}}<\overline{\boldsymbol{\mu}}<1$ for $\frac{\gamma}{\gamma+1}>\widehat{\boldsymbol{\mu}} \geq \frac{\gamma}{n}$. Then, $\operatorname{Pr}\{\underline{\boldsymbol{\mu}}<\mu<\overline{\boldsymbol{\mu}}\} \geq 1-\delta$.

\section{Conclusion}

In this paper, we have established a general theory of truncated inverse sampling for estimating the mean value of a large class of random variables. We have applied such a theory to the common important variables such as binomial, Poisson, hyper-geometrical, and bounded variables. Rigorous methods have been derived for determining the thresholds and maximum sample sizes to ensure statistical accuracy. Interval estimation methods have also been developed. 


\section{A Proof of Theorem 1}

The theorem can be shown by establishing Lemmas 1 to 4 as follows.

Lemma 1 Suppose $z \geq \frac{\gamma}{n}$. Then, $\operatorname{Pr}\{\widehat{\boldsymbol{\mu}} \leq z\}=\operatorname{Pr}\left\{\sum_{i=1}^{m} X_{i}<\gamma\right\}$ where $m=\left\lceil\frac{\gamma}{z}\right\rceil-1$.

Proof. By the assumption that $z \geq \frac{\gamma}{n}$ and the definition of the sampling scheme,

$$
\{\widehat{\boldsymbol{\mu}}>z, \mathbf{k}<\gamma\}=\left\{\frac{\mathbf{k}}{\mathbf{n}}>z, \mathbf{k}<\gamma\right\} \subseteq\left\{\frac{\mathbf{k}}{\mathbf{n}}>\frac{\gamma}{n}, \mathbf{k}<\gamma\right\}=\left\{\frac{\mathbf{k}}{\mathbf{n}}>\frac{\gamma}{n}, \mathbf{k}<\gamma, \mathbf{n}=n\right\}=\emptyset .
$$

Therefore, $\{\widehat{\boldsymbol{\mu}}>z\}=\{\widehat{\boldsymbol{\mu}}>z, \mathbf{k}<\gamma\} \cup\{\widehat{\boldsymbol{\mu}}>z, \mathbf{k} \geq \gamma\}=\{\widehat{\boldsymbol{\mu}}>z, \mathbf{k} \geq \gamma\}=\left\{\frac{\gamma}{\mathbf{n}}>z, \mathbf{k} \geq \gamma\right\}$. To show the lemma, it remains to show that $\left\{\frac{\gamma}{\mathbf{n}}>z, \mathbf{k} \geq \gamma\right\}=\operatorname{Pr}\left\{\sum_{i=1}^{m} X_{i} \geq \gamma\right\}$. Since all $X_{i}$ are non-negative, we have $\left\{\frac{\gamma}{\mathbf{n}}>z, \mathbf{k} \geq \gamma\right\}=\{\mathbf{n} \leq m, \mathbf{k} \geq \gamma\} \subseteq\left\{\mathbf{n} \leq m, \sum_{i=1}^{m} X_{i} \geq \gamma\right\} \subseteq\left\{\sum_{i=1}^{m} X_{i} \geq \gamma\right\}$. On the other hand, by the assumption that $z \geq \frac{\gamma}{n}$, we have $m=\left\lceil\frac{\gamma}{z}\right\rceil-1 \leq n-1$. Hence, by the definition of the sampling scheme, we have $\left\{\sum_{i=1}^{m} X_{i} \geq \gamma\right\} \subseteq\left\{\mathbf{n} \leq m, \mathbf{k} \geq \gamma, \sum_{i=1}^{m} X_{i} \geq \gamma\right\} \subseteq\{\mathbf{n} \leq$ $m, \mathbf{k} \geq \gamma\}$. It follows that $\left\{\sum_{i=1}^{m} X_{i} \geq \gamma\right\}=\{\mathbf{n} \leq m, \mathbf{k} \geq \gamma\}=\left\{\frac{\gamma}{\mathbf{n}}>z, \mathbf{k} \geq \gamma\right\}=\{\widehat{\boldsymbol{\mu}}>z\}$. This completes the proof of the lemma.

Lemma 2 Suppose $z<\frac{\gamma}{n}$. Then, $\operatorname{Pr}\{\widehat{\boldsymbol{\mu}} \leq z\}=\operatorname{Pr}\left\{\frac{\sum_{i=1}^{n} X_{i}}{n} \leq z\right\}$.

Proof. By the assumption that $z<\frac{\gamma}{n}$ and the definition of the sampling scheme,

$$
\{\widehat{\boldsymbol{\mu}} \leq z, \mathbf{k} \geq \gamma\}=\left\{\frac{\gamma}{\mathbf{n}} \leq z, \mathbf{k} \geq \gamma\right\} \subseteq\left\{\frac{\gamma}{\mathbf{n}}<\frac{\gamma}{n}, \mathbf{k} \geq \gamma\right\}=\{\mathbf{n}>n, \mathbf{k} \geq \gamma\}=\emptyset .
$$

Therefore,

$$
\begin{aligned}
\{\widehat{\boldsymbol{\mu}} \leq z\} & =\{\widehat{\boldsymbol{\mu}} \leq z, \mathbf{k}<\gamma\} \cup\{\widehat{\boldsymbol{\mu}} \leq z, \mathbf{k} \geq \gamma\}=\{\widehat{\boldsymbol{\mu}} \leq z, \mathbf{k}<\gamma\}=\left\{\frac{\mathbf{k}}{\mathbf{n}} \leq z, \mathbf{k}<\gamma\right\} \\
& =\left\{\frac{\mathbf{k}}{\mathbf{n}} \leq z, \mathbf{k}<\gamma, \mathbf{n}=n\right\}=\left\{\frac{\sum_{i=1}^{n} X_{i}}{n} \leq z, \sum_{i=1}^{n} X_{i}<\gamma, \mathbf{n}=n\right\} \subseteq\left\{\frac{\sum_{i=1}^{n} X_{i}}{n} \leq z\right\} .
\end{aligned}
$$

On the other hand, by the definition of the sampling scheme and the assumption that $z<\frac{\gamma}{n}$, we have $\left\{\frac{\sum_{i=1}^{n} X_{i}}{n} \leq z\right\} \subseteq\left\{\frac{\sum_{i=1}^{n} X_{i}}{n} \leq z, \mathbf{n}=n\right\}=\left\{\frac{\sum_{i=1}^{n} X_{i}}{n} \leq z, \sum_{i=1}^{n} X_{i}<\gamma, \mathbf{n}=n\right\}=\{\widehat{\boldsymbol{\mu}} \leq z\}$. It follows that $\{\widehat{\boldsymbol{\mu}} \leq z\}=\left\{\frac{\sum_{i=1}^{n} X_{i}}{n} \leq z\right\}$. This completes the proof of the lemma.

Lemma 3 Suppose $z \geq \frac{\gamma}{n}$. Then, $\operatorname{Pr}\{\widehat{\boldsymbol{\mu}} \geq z\}=\operatorname{Pr}\left\{\sum_{i=1}^{m} X_{i} \geq \gamma\right\}$ where $m=\left\lfloor\frac{\gamma}{z}\right\rfloor$. 
Proof. By the assumption that $z \geq \frac{\gamma}{n}$ and the definition of the sampling scheme,

$$
\{\widehat{\boldsymbol{\mu}} \geq z, \mathbf{k}<\gamma\}=\left\{\frac{\mathbf{k}}{\mathbf{n}} \geq z, \mathbf{k}<\gamma\right\}=\left\{\frac{\mathbf{k}}{\mathbf{n}} \geq z, \mathbf{k}<\gamma, \mathbf{n}=n\right\}=\emptyset .
$$

Therefore, $\{\widehat{\boldsymbol{\mu}} \geq z\}=\{\widehat{\boldsymbol{\mu}} \geq z, \mathbf{k}<\gamma\} \cup\{\widehat{\boldsymbol{\mu}} \geq z, \mathbf{k} \geq \gamma\}=\{\widehat{\boldsymbol{\mu}} \geq z, \mathbf{k} \geq \gamma\}=\left\{\frac{\gamma}{\mathbf{n}} \geq z, \mathbf{k} \geq \gamma\right\}$. To show the lemma, it remains to show that $\left\{\frac{\gamma}{\mathbf{n}} \geq z, \mathbf{k} \geq \gamma\right\}=\operatorname{Pr}\left\{\sum_{i=1}^{m} X_{i} \geq \gamma\right\}$. Since all $X_{i}$ are non-negative, we have $\left\{\frac{\gamma}{\mathbf{n}} \geq z, \mathbf{k} \geq \gamma\right\}=\{\mathbf{n} \leq m, \mathbf{k} \geq \gamma\} \subseteq\left\{\mathbf{n} \leq m, \sum_{i=1}^{m} X_{i} \geq \gamma\right\} \subseteq\left\{\sum_{i=1}^{m} X_{i} \geq \gamma\right\}$. On the other hand, by the assumption that $z \geq \frac{\gamma}{n}$, we have $m=\left\lfloor\frac{\gamma}{z}\right\rfloor \leq n$. By the definition of the sampling scheme and the fact that all $X_{i}$ are non-negative, $\left\{\sum_{i=1}^{m} X_{i} \geq \gamma\right\} \subseteq\left\{\mathbf{n} \leq m, \sum_{i=1}^{m} X_{i} \geq\right.$ $\gamma\} \subseteq\{\mathbf{n} \leq m, \mathbf{k} \geq \gamma\}$. Hence, $\left\{\sum_{i=1}^{m} X_{i} \geq \gamma\right\}=\{\mathbf{n} \leq m, \mathbf{k} \geq \gamma\}=\left\{\frac{\gamma}{\mathbf{n}} \geq z, \mathbf{k} \geq \gamma\right\}=\{\widehat{\boldsymbol{\mu}} \geq z\}$. This completes the proof of the lemma.

Lemma 4 Suppose $z<\frac{\gamma}{n}$. Then, $\operatorname{Pr}\{\widehat{\boldsymbol{\mu}} \geq z\}=\operatorname{Pr}\left\{\frac{\sum_{i=1}^{n} X_{i}}{n} \geq z\right\}$.

Proof. By the assumption that $z<\frac{\gamma}{n}$ and the definition of the sampling scheme,

$$
\{\widehat{\boldsymbol{\mu}}<z, \mathbf{k} \geq \gamma\}=\left\{\frac{\gamma}{\mathbf{n}}<z, \mathbf{k} \geq \gamma\right\} \subseteq\left\{\frac{\gamma}{\mathbf{n}}<\frac{\gamma}{n}, \mathbf{k} \geq \gamma\right\}=\{\mathbf{n}>n, \mathbf{k} \geq \gamma\}=\emptyset .
$$

Therefore,

$$
\begin{aligned}
\{\widehat{\boldsymbol{\mu}}<z\} & =\{\widehat{\boldsymbol{\mu}}<z, \mathbf{k}<\gamma\} \cup\{\widehat{\boldsymbol{\mu}}<z, \mathbf{k} \geq \gamma\}=\{\widehat{\boldsymbol{\mu}}<z, \mathbf{k}<\gamma\}=\left\{\frac{\mathbf{k}}{\mathbf{n}}<z, \mathbf{k}<\gamma\right\} \\
& =\left\{\frac{\mathbf{k}}{\mathbf{n}}<z, \mathbf{k}<\gamma, \mathbf{n}=n\right\}=\left\{\frac{\sum_{i=1}^{n} X_{i}}{n}<z, \sum_{i=1}^{n} X_{i}<\gamma, \mathbf{n}=n\right\} \subseteq\left\{\frac{\sum_{i=1}^{n} X_{i}}{n}<z\right\} .
\end{aligned}
$$

On the other hand, by the definition of the sampling scheme and the assumption that $z<\frac{\gamma}{n}$,

$$
\left\{\frac{\sum_{i=1}^{n} X_{i}}{n}<z\right\} \subseteq\left\{\frac{\sum_{i=1}^{n} X_{i}}{n}<z, \mathbf{n}=n\right\}=\left\{\frac{\sum_{i=1}^{n} X_{i}}{n}<z, \sum_{i=1}^{n} X_{i}<\gamma, \mathbf{n}=n\right\}=\{\widehat{\boldsymbol{\mu}}<z\} .
$$

It follows that $\{\widehat{\boldsymbol{\mu}}<z\}=\left\{\frac{\sum_{i=1}^{n} X_{i}}{n}<z\right\}$, i.e., $\{\widehat{\boldsymbol{\mu}} \geq z\}=\left\{\frac{\sum_{i=1}^{n} X_{i}}{n} \geq z\right\}$. This completes the proof of the lemma.

\section{B Proof of Theorem 2}

By the definition of the truncated inverse sampling scheme,

$$
\begin{aligned}
\mathbb{E}[\mathbf{n}] & =n \operatorname{Pr}\left\{\sum_{i=1}^{n} X_{i}<\gamma\right\}+\sum_{m=1}^{n} m \operatorname{Pr}\left\{\sum_{i=1}^{m-1} X_{i}<\gamma, \sum_{i=1}^{m} X_{i} \geq \gamma\right\} \\
& <n \operatorname{Pr}\left\{\sum_{i=1}^{n} X_{i}<\gamma\right\}+\sum_{m=1}^{n} n \operatorname{Pr}\left\{\sum_{i=1}^{m-1} X_{i}<\gamma, \sum_{i=1}^{m} X_{i} \geq \gamma\right\}=n .
\end{aligned}
$$


By the fact that $X_{i}$ is non-negative,

$$
\left(\bigcup_{m=n+1}^{\infty}\left\{\sum_{i=1}^{m-1} X_{i}<\gamma, \sum_{i=1}^{m} X_{i} \geq \gamma\right\}\right) \bigcup\left\{\sum_{i=1}^{\infty} X_{i}<\gamma\right\}=\left\{\sum_{i=1}^{n} X_{i}<\gamma\right\} .
$$

Since $\mathbb{E}[X]=\mu$ is positive and the corresponding variance $\sigma^{2}$ is finite, we have, by Chebyshev's inequality,

$$
\begin{aligned}
0 \leq \operatorname{Pr}\left\{\sum_{i=1}^{\infty} X_{i}<\gamma\right\} & =\lim _{k \rightarrow \infty} \operatorname{Pr}\left\{\sum_{i=1}^{k} X_{i}<\gamma\right\}=\lim _{k \rightarrow \infty} \operatorname{Pr}\left\{\frac{\sum_{i=1}^{k} X_{i}}{k}-\mu<\frac{\gamma}{k}-\mu\right\} \\
& \leq \lim _{k \rightarrow \infty} \operatorname{Pr}\left\{\left|\frac{\sum_{i=1}^{k} X_{i}}{k}-\mu\right|>\left|\frac{\gamma}{k}-\mu\right|\right\} \leq \lim _{k \rightarrow \infty} \frac{\frac{\sigma^{2}}{k}}{\left|\frac{\gamma}{k}-\mu\right|^{2}}=0 .
\end{aligned}
$$

Hence,

$$
\begin{aligned}
\mathbb{E}[\mathbf{n}] & =\sum_{m=n+1}^{\infty} n \operatorname{Pr}\left\{\sum_{i=1}^{m-1} X_{i}<\gamma, \sum_{i=1}^{m} X_{i} \geq \gamma\right\}+\sum_{m=1}^{n} m \operatorname{Pr}\left\{\sum_{i=1}^{m-1} X_{i}<\gamma, \sum_{i=1}^{m} X_{i} \geq \gamma\right\} \\
& <\sum_{m=1}^{\infty} m \operatorname{Pr}\left\{\sum_{i=1}^{m-1} X_{i}<\gamma, \sum_{i=1}^{m} X_{i} \geq \gamma\right\}=\mathbb{E}[\mathbf{m}],
\end{aligned}
$$

where $\mathbf{m}$ is the sample number of the classical inverse sampling scheme with the following stopping rule: Sampling is continued until the sample sum is no less than $\gamma$. By the definition of the classical inverse sampling, we have $\sum_{i=1}^{\mathbf{m}-1} X_{i}<\gamma$. Applying Wald's equation, we have $\mathbb{E}\left[\sum_{i=1}^{\mathbf{m}-1} X_{i}\right]=\mathbb{E}[\mathbf{m}-1] \mathbb{E}[X]<\gamma$, which implies that $\mathbb{E}[\mathbf{m}]<\frac{\gamma}{\mathbb{E}[X]}+1=\frac{\gamma}{\mu}+1$. Since $\mathbb{E}[\mathbf{n}]$ is less than both $n$ and $\mathbb{E}[\mathbf{m}]$ as shown above, we have $\mathbb{E}[\mathbf{n}]<\min \left\{n, \frac{\gamma}{\mu}+1\right\}$.

In the special case that $\gamma$ is a positive integer and that $X$ is a Bernoulli random variable such that $\mathbb{E}[X]=p \in(0,1)$, we have $\sum_{i=1}^{\mathbf{m}} X_{i}=\gamma$ and consequently, by Wald's equation, $\mathbb{E}\left[\sum_{i=1}^{\mathbf{m}} X_{i}\right]=$ $\mathbb{E}[\mathbf{m}] \mathbb{E}[X]=\gamma$, from which we get $\mathbb{E}[\mathbf{m}]=\frac{\gamma}{\mathbb{E}[X]}=\frac{\gamma}{p}$ and it follows that $\mathbb{E}[\mathbf{n}]<\min \left\{n, \frac{\gamma}{p}\right\}$. This completes the proof the theorem.

\section{Proof of Theorem 3}

We need some preliminary results. The following lemma is a slight modification of Hoeffding [7].

Lemma 5 Let $X_{1}, \cdots, X_{n}$ be i.i.d. random variables bounded in $[0,1]$ with common mean value $\mu \in(0,1)$. Then, $\operatorname{Pr}\left\{\frac{\sum_{i=1}^{n} X_{i}}{n} \geq z\right\} \leq \exp \left(n \mathscr{M}_{\mathrm{B}}(z, \mu)\right)$ for $1 \geq z \geq \mu$. Similarly, $\operatorname{Pr}\left\{\frac{\sum_{i=1}^{n} X_{i}}{n} \leq z\right\} \leq$ $\exp \left(n \mathscr{M}_{\mathrm{B}}(z, \mu)\right)$ for $0 \leq z \leq \mu$.

Proof. For $z=\mu$, we have $\operatorname{Pr}\left\{\frac{\sum_{i=1}^{n} X_{i}}{n} \geq z\right\} \leq \exp \left(n \mathscr{M}_{\mathrm{B}}(z, \mu)\right)=1$. For $\mu<z<1$, it was shown by Hoeffding in [7] that $\operatorname{Pr}\left\{\frac{\sum_{i=1}^{n} X_{i}}{n} \geq z\right\} \leq \exp \left(n \mathscr{M}_{\mathrm{B}}(z, \mu)\right)$. For $z=1$, we have $\operatorname{Pr}\left\{\frac{\sum_{i=1}^{n} X_{i}}{n} \geq z\right\}=$ $\prod_{i=1}^{n} \operatorname{Pr}\left\{X_{i}=1\right\} \leq \prod_{i=1}^{n} \mathbb{E}\left[X_{i}\right]=\mu^{n}=\exp \left(n \mathscr{M}_{\mathrm{B}}(1, \mu)\right)$.

For $z=0$, we have $\operatorname{Pr}\left\{\frac{\sum_{i=1}^{n} X_{i}}{n} \leq z\right\}=\prod_{i=1}^{n}\left(1-\operatorname{Pr}\left\{X_{i} \neq 0\right\}\right) \leq \prod_{i=1}^{n}\left(1-\mathbb{E}\left[X_{i}\right]\right)=(1-\mu)^{n}=$ $\exp \left(n \mathscr{M}_{\mathrm{B}}(0, \mu)\right)$. For $0<z<\mu$, it was shown by Hoeffding in [7] that $\operatorname{Pr}\left\{\frac{\sum_{i=1}^{n} X_{i}}{n} \leq z\right\} \leq$ $\exp \left(n \mathscr{M}_{\mathrm{B}}(z, \mu)\right)$. For $z=\mu$, we have $\operatorname{Pr}\left\{\frac{\sum_{i=1}^{n} X_{i}}{n} \leq z\right\} \leq \exp \left(n \mathscr{M}_{\mathrm{B}}(z, \mu)\right)=1$. 
Lemma 6 Let $0<\varepsilon<1$. Then, $\mathscr{M}_{\mathrm{I}}(\mu+\varepsilon \mu, \mu)$ is monotonically decreasing with respect to $\mu \in\left(0, \frac{1}{1+\varepsilon}\right)$. Similarly, $\mathscr{M}_{\mathrm{I}}(\mu-\varepsilon \mu, \mu)$ is monotonically decreasing with respect to $\mu \in(0,1)$.

Proof. Note that $\frac{\partial \mathscr{M}_{\mathrm{I}}(\mu+\varepsilon \mu, \mu)}{\partial \mu}=-\frac{1}{\mu^{2}(1+\varepsilon)} \ln \left[\frac{1-\mu}{1-\mu(1+\varepsilon)}\right]+\frac{\varepsilon}{\mu(1-\mu)(1+\varepsilon)} \leq 0$ if $\ln \left[\frac{1-\mu}{1-\mu(1+\varepsilon)}\right] \geq \frac{\varepsilon \mu}{1-\mu}$, i.e.,

$$
\ln \left(1-\frac{\varepsilon \mu}{1-\mu}\right) \leq-\frac{\varepsilon \mu}{1-\mu}
$$

As a consequence of $0<\mu<\frac{1}{1+\varepsilon}$, we have $0<\frac{\varepsilon \mu}{1-\mu}<1$. Since $\ln (1-x)<-x$ for any $x \in(0,1)$, it follows that (10) holds and thus $\mathscr{M}_{\mathrm{I}}(\mu+\varepsilon \mu, \mu)$ is monotonically decreasing with respect to $\mu \in\left(0, \frac{1}{1+\varepsilon}\right)$.

Similarly, to show that $\mathscr{M}_{\mathrm{I}}(\mu-\varepsilon \mu, \mu)$ is monotonically decreasing with respect to $\mu$, note that $\frac{\partial \mathscr{M}_{\mathrm{I}}(\mu-\varepsilon \mu, \mu)}{\partial \mu}=-\frac{1}{\mu^{2}(1-\varepsilon)} \ln \left[\frac{1-\mu}{1-\mu(1-\varepsilon)}\right]-\frac{\varepsilon}{\mu(1-\mu)(1-\varepsilon)} \leq 0$ if $\ln \left[\frac{1-\mu}{1-\mu(1-\varepsilon)}\right] \geq-\frac{\varepsilon \mu}{1-\mu}$, i.e.,

$$
\ln \left(1+\frac{\varepsilon \mu}{1-\mu}\right) \leq \frac{\varepsilon \mu}{1-\mu} .
$$

Since $\frac{\varepsilon \mu}{1-\mu}>0$ and $\ln (1+x)<x$ for any $x \in(0, \infty)$, we have that (11) holds and thus $\mathscr{M}_{\mathrm{I}}(\mu-\varepsilon \mu, \mu)$ is monotonically decreasing with respect to $\mu \in(0,1)$.

Lemma $7 \mathscr{M}_{\mathrm{I}}(\mu+\varepsilon \mu, \mu)>\mathscr{M}_{\mathrm{I}}(\mu-\varepsilon \mu, \mu)$ for $\mu \in\left(0, \frac{1}{2}\right)$ and $0<\varepsilon<1$.

Proof. Direct computation shows that

$$
\frac{\partial \mathscr{M}_{\mathrm{I}}(\mu+\varepsilon \mu, \mu)}{\partial \varepsilon}=-\frac{1}{(1+\varepsilon)^{2} \mu} \ln \left[\frac{1-\mu}{1-(1+\varepsilon) \mu}\right], \quad \frac{\partial \mathscr{M}_{\mathrm{I}}(\mu-\varepsilon \mu, \mu)}{\partial \varepsilon}=\frac{1}{(1-\varepsilon)^{2} \mu} \ln \left[\frac{1-\mu}{1-(1-\varepsilon) \mu}\right] .
$$

Since $\ln \left[\frac{1-\mu}{1-(1+\varepsilon) \mu}\right]<\frac{\varepsilon \mu}{1-(1+\varepsilon) \mu}$ and $\ln \left[\frac{1-\mu}{1-(1-\varepsilon) \mu}\right]<-\frac{\varepsilon \mu}{1-(1-\varepsilon) \mu}$, we have

$$
\frac{\partial \mathscr{M}_{\mathrm{I}}(\mu+\varepsilon \mu, \mu)}{\partial \varepsilon}-\frac{\partial \mathscr{M}_{\mathrm{I}}(\mu-\varepsilon \mu, \mu)}{\partial \varepsilon}>-\frac{1}{(1+\varepsilon)^{2} \mu} \frac{\varepsilon \mu}{1-(1+\varepsilon) \mu}+\frac{1}{(1-\varepsilon)^{2} \mu} \frac{\varepsilon \mu}{1-(1-\varepsilon) \mu}>0
$$

if $(1+\varepsilon)^{2}[1-(1+\varepsilon) \mu]-(1-\varepsilon)^{2}[1-(1-\varepsilon) \mu]>0$, or equivalently, $4 \varepsilon-2 \varepsilon\left(3+\varepsilon^{2}\right) \mu>0$, which is true because $4 \varepsilon-2 \varepsilon\left(3+\varepsilon^{2}\right) \mu>4 \varepsilon-2 \varepsilon(3+1) \times \frac{1}{2}=0$ as a result of $0<\varepsilon<1$ and $0<\mu<\frac{1}{2}$. The lemma immediately follows from the fact that $\frac{\partial \mathscr{M}_{\mathrm{I}}(\mu+\varepsilon \mu, \mu)}{\partial \varepsilon}$ is greater than $\frac{\partial \mathscr{M}_{\mathrm{I}}(\mu-\varepsilon \mu, \mu)}{\partial \varepsilon}$ for $0<\varepsilon<1,0<\mu<\frac{1}{2}$ and $\mathscr{M}_{\mathrm{I}}(\mu+\varepsilon \mu, \mu)$ is equal to $\mathscr{M}_{\mathrm{I}}(\mu-\varepsilon \mu, \mu)$ for $\varepsilon=0$.

Lemma 8 Let $0<\varepsilon<\frac{1}{2}$. Then, $\mathscr{M}_{\mathrm{I}}(\mu+\varepsilon, \mu)$ is monotonically increasing with respect to $\mu \in\left(0, \frac{1}{2}-\varepsilon\right)$. Similarly, $\mathscr{M}_{\mathrm{I}}(\mu-\varepsilon, \mu)$ is monotonically increasing with respect to $\mu \in\left(\varepsilon, \frac{1}{2}+\varepsilon\right)$. 
Proof. It can be shown that $\frac{\mathscr{M}_{\mathrm{I}}(\mu+\varepsilon, \mu)}{\partial \mu}=-\frac{1}{(\mu+\varepsilon)^{2}} \ln \left(\frac{1-\mu}{1-\mu-\varepsilon}\right)+\frac{\varepsilon}{\mu(\mu+\varepsilon)(1-\mu)}>0$ if $\ln \left(\frac{1-\mu}{1-\mu-\varepsilon}\right)<$ $\frac{\varepsilon(\mu+\varepsilon)}{\mu(1-\mu)}$. Since $\ln \left(\frac{1-\mu}{1-\mu-\varepsilon}\right)<\frac{\varepsilon}{1-\mu-\varepsilon}$, it suffices to have $\frac{\varepsilon}{1-\mu-\varepsilon}<\frac{\varepsilon(\mu+\varepsilon)}{\mu(1-\mu)}$, or equivalently, $\mu(1-\mu)<$ $(1-\mu-\varepsilon)(\mu+\varepsilon)=\mu(1-\mu)-\varepsilon \mu+(1-\mu) \varepsilon-\varepsilon^{2}$, which can be ensured by $0<\mu<\frac{1}{2}-\varepsilon$. This proves the first statement of the lemma.

Similarly, $\frac{\mathscr{M}_{\mathrm{I}}(\mu-\varepsilon, \mu)}{\partial \mu}=-\frac{1}{(\mu-\varepsilon)^{2}} \ln \left(\frac{1-\mu}{1-\mu+\varepsilon}\right)-\frac{\varepsilon}{\mu(\mu-\varepsilon)(1-\mu)}>0$ if $\ln \left(\frac{1-\mu}{1-\mu+\varepsilon}\right)<-\frac{\varepsilon(\mu-\varepsilon)}{\mu(1-\mu)}$. Since $\ln \left(\frac{1-\mu}{1-\mu+\varepsilon}\right)<-\frac{\varepsilon}{1-\mu+\varepsilon}$, to ensure $\frac{\mathscr{M}_{\mathrm{I}}(\mu-\varepsilon, \mu)}{\partial \mu}>0$, it suffices to have $-\frac{\varepsilon}{1-\mu+\varepsilon}<-\frac{\varepsilon(\mu-\varepsilon)}{\mu(1-\mu)}$, or equivalently, $\mu(1-\mu)>(1-\mu+\varepsilon)(\mu-\varepsilon)=\mu(1-\mu)+\varepsilon \mu-\varepsilon(1-\mu)-\varepsilon^{2}$, which can be guaranteed by $\varepsilon<\mu<\frac{1}{2}+\frac{\varepsilon}{2}$. This proves the second statement of the lemma.

Lemma $9 \mathscr{M}_{\mathrm{I}}(\mu+\varepsilon, \mu)>\mathscr{M}_{\mathrm{I}}(\mu-\varepsilon, \mu)$ for $0<\varepsilon<\mu<\frac{1}{2}$.

Proof. It can be verified that $\frac{\partial \mathscr{M}_{\mathrm{I}}(\mu+\varepsilon, \mu)}{\partial \varepsilon}=-\frac{1}{(\mu+\varepsilon)^{2}} \ln \left(\frac{1-\mu}{1-\mu-\varepsilon}\right)$ and $\frac{\partial \mathscr{M}_{\mathrm{I}}(\mu-\varepsilon, \mu)}{\partial \varepsilon}=\frac{1}{(\mu-\varepsilon)^{2}} \ln \left(\frac{1-\mu}{1-\mu+\varepsilon}\right)$. Since $\ln \left(\frac{1-\mu}{1-\mu-\varepsilon}\right)<\frac{\varepsilon}{1-\mu-\varepsilon}$ and $\ln \left(\frac{1-\mu}{1-\mu+\varepsilon}\right)<-\frac{\varepsilon}{1-\mu+\varepsilon}$, to ensure $\frac{\partial \mathscr{M}_{\mathrm{I}}(\mu+\varepsilon, \mu)}{\partial \varepsilon}>\frac{\partial \mathscr{M}_{\mathrm{I}}(\mu-\varepsilon, \mu)}{\partial \varepsilon}$, it suffices to have $-\frac{1}{(\mu+\varepsilon)^{2}} \frac{\varepsilon}{1-(\mu+\varepsilon)}>-\frac{1}{(\mu-\varepsilon)^{2}} \frac{\varepsilon}{1-\mu+\varepsilon}$, or equivalently, $2 \mu-3 \mu^{2}>\varepsilon^{2}$, which is true because $2 \mu-3 \mu^{2}-\varepsilon^{2}>2 \mu-3 \mu^{2}-\mu^{2}=2 \mu(1-2 \mu)>0$ as a result of $0<\varepsilon<\mu<\frac{1}{2}$. Therefore, the lemma is true since $\mathscr{M}_{\mathrm{I}}(\mu+\varepsilon, \mu)=\mathscr{M}_{\mathrm{I}}(\mu-\varepsilon, \mu)$ for $\varepsilon=0$ and $\frac{\partial \mathscr{M}_{\mathrm{I}}(\mu+\varepsilon, \mu)}{\partial \varepsilon}>\frac{\partial \mathscr{M}_{\mathrm{I}}(\mu-\varepsilon, \mu)}{\partial \varepsilon}$ for $0<\varepsilon<\mu<\frac{1}{2}$. This completes the proof of the lemma.

Lemma 10 Let $0<\varepsilon<\frac{1}{2}$. Then, $\mathscr{M}_{\mathrm{B}}(\mu+\varepsilon, \mu)$ is monotonically increasing with respect to $\mu \in\left(0, \frac{1}{2}-\varepsilon\right)$. Similarly, $\mathscr{M}_{\mathrm{B}}(\mu-\varepsilon, \mu)$ is monotonically increasing with respect to $\mu \in\left(\varepsilon, \frac{1}{2}\right)$.

Proof. Our computation shows that

$$
\frac{\partial \mathscr{M}_{\mathrm{B}}(\mu+\varepsilon, \mu)}{\partial \mu}=\ln \frac{\mu(1-\mu-\varepsilon)}{(\mu+\varepsilon)(1-\mu)}+\frac{\varepsilon}{\mu(1-\mu)}, \quad \frac{\partial^{2} \mathscr{M}_{\mathrm{B}}(\mu+\varepsilon, \mu)}{\partial \mu \partial \varepsilon}=\frac{1}{\mu(1-\mu)}-\frac{1}{(\mu+\varepsilon)(1-\mu-\varepsilon)} .
$$

Since $\frac{\partial \mathscr{M}_{\mathrm{B}}(\mu+\varepsilon, \mu)}{\partial \mu}=0$ for $\varepsilon=0$ and $\frac{\partial^{2} \mathscr{M}_{\mathrm{B}}(\mu+\varepsilon, \mu)}{\partial \mu \partial \varepsilon}>0$ for $\varepsilon<\frac{1}{2}-\mu$, it must be true that $\frac{\partial \mathscr{M}_{\mathrm{B}}(\mu+\varepsilon, \mu)}{\partial \mu}>0$ for $\mu \in\left(0, \frac{1}{2}-\varepsilon\right)$. This proves the first statement of the lemma. Similarly, we can show that $\frac{\partial \mathscr{M}_{\mathrm{B}}(\mu-\varepsilon, \mu)}{\partial \mu}=\ln \frac{\mu(1-\mu+\varepsilon)}{(\mu-\varepsilon)(1-\mu)}-\frac{\varepsilon}{\mu(1-\mu)}$ and $\frac{\partial^{2} \mathscr{M}_{\mathrm{B}}(\mu-\varepsilon, \mu)}{\partial \mu \partial \varepsilon}=\frac{1}{(\mu-\varepsilon)(1-\mu+\varepsilon)}-\frac{1}{\mu(1-\mu)}$. Since $\frac{\partial \mathscr{M}_{\mathrm{B}}(\mu-\varepsilon, \mu)}{\partial \mu}=0$ for $\varepsilon=0$ and $\frac{\partial^{2} \mathscr{M}_{\mathrm{B}}(\mu-\varepsilon, \mu)}{\partial \mu \partial \varepsilon}>0$ for $0<\varepsilon<\mu<\frac{1}{2}$, it must be true that $\frac{\partial \mathscr{M}_{\mathrm{B}}(\mu-\varepsilon, \mu)}{\partial \mu}>0$ for $0<\varepsilon<\mu<\frac{1}{2}$. This proves the second statement of the lemma.

Lemma 11 Let $0<\varepsilon<\frac{1}{2}$. Then, $\mathscr{M}_{\mathrm{B}}(\mu+\varepsilon, \mu)>\mathscr{M}_{\mathrm{B}}(\mu-\varepsilon, \mu)$ for $\mu \in\left(\varepsilon, \frac{1}{2}\right)$. 
Proof. Straightforward computation shows that

$$
\frac{\partial \mathscr{M}_{\mathrm{B}}(\mu+\varepsilon, \mu)}{\partial \varepsilon}=\ln \left(\frac{\mu}{1-\mu} \frac{1-\mu-\varepsilon}{\mu+\varepsilon}\right), \quad \frac{\partial \mathscr{M}_{\mathrm{B}}(\mu-\varepsilon, \mu)}{\partial \varepsilon}=-\ln \left(\frac{\mu}{1-\mu} \frac{1-\mu+\varepsilon}{\mu-\varepsilon}\right) .
$$

Thus, $\frac{\partial \mathscr{M}_{\mathrm{B}}(\mu+\varepsilon, \mu)}{\partial \varepsilon}-\frac{\partial \mathscr{M}_{\mathrm{B}}(\mu-\varepsilon, \mu)}{\partial \varepsilon}=\ln \frac{\mu^{2}}{(1-\mu)^{2}} \frac{(1-\mu)^{2}-\varepsilon^{2}}{\mu^{2}-\varepsilon^{2}}>0$ if $\varepsilon<\mu<\frac{1}{2}$. By virtue of such result and the fact that $\mathscr{M}_{\mathrm{B}}(\mu+\varepsilon, \mu)=\mathscr{M}_{\mathrm{B}}(\mu-\varepsilon, \mu)$ for $\varepsilon=0$, we have $\mathscr{M}_{\mathrm{B}}(\mu+\varepsilon, \mu)>\mathscr{M}_{\mathrm{B}}(\mu-\varepsilon, \mu)$ for $\varepsilon<\mu<\frac{1}{2}$. This proves the lemma.

Lemma 12 Let $0<\varepsilon<1$. Then, $\mathscr{M}_{\mathrm{B}}(\mu+\varepsilon \mu, \mu)$ is monotonically decreasing with respect to $\mu \in\left(0, \frac{1}{1+\varepsilon}\right)$. Similarly, $\mathscr{M}_{\mathrm{B}}(\mu-\varepsilon \mu, \mu)$ is monotonically decreasing with respect to $\mu \in(0,1)$.

Proof. The first statement of the lemma is true because

$\frac{\partial \mathscr{M}_{\mathrm{B}}(\mu+\varepsilon \mu, \mu)}{\partial \mu}=(1+\varepsilon) \ln \left[1-\frac{\varepsilon}{(1+\varepsilon)(1-\mu)}\right]+\frac{\varepsilon}{1-\mu}<(1+\varepsilon) \times\left[-\frac{\varepsilon}{(1+\varepsilon)(1-\mu)}\right]+\frac{\varepsilon}{1-\mu}=0$

for $0<\mu<\frac{1}{1+\varepsilon}$. Similarly, the second statement of the lemma is true because

$\frac{\partial \mathscr{M}_{\mathrm{B}}(\mu-\varepsilon \mu, \mu)}{\partial \mu}=(1-\varepsilon) \ln \left[1+\frac{\varepsilon}{(1-\varepsilon)(1-\mu)}\right]-\frac{\varepsilon}{1-\mu}<(1-\varepsilon) \times\left[\frac{\varepsilon}{(1-\varepsilon)(1-\mu)}\right]-\frac{\varepsilon}{1-\mu}=0$

for $0<\mu<1$.

Lemma 13 Let $0<\varepsilon<1$. Then, $\mathscr{M}_{\mathrm{B}}(\mu+\varepsilon \mu, \mu)>\mathscr{M}_{\mathrm{B}}(\mu-\varepsilon \mu, \mu)$ for $\mu \in\left(0, \frac{1}{2}\right)$.

Proof. It can be shown by tedious computation that

$$
\frac{\partial \mathscr{M}_{\mathrm{B}}(\mu+\varepsilon \mu, \mu)}{\partial \varepsilon}=\mu \ln \frac{1-\mu-\varepsilon \mu}{(1+\varepsilon)(1-\mu)}, \quad \frac{\partial \mathscr{M}_{\mathrm{B}}(\mu-\varepsilon \mu, \mu)}{\partial \varepsilon}=-\mu \ln \frac{1-\mu+\varepsilon \mu}{(1-\varepsilon)(1-\mu)} .
$$

Hence,

$$
\frac{\partial \mathscr{M}_{\mathrm{B}}(\mu+\varepsilon \mu, \mu)}{\partial \varepsilon}-\frac{\partial \mathscr{M}_{\mathrm{B}}(\mu-\varepsilon \mu, \mu)}{\partial \varepsilon}=\mu \ln \left[\frac{1-\mu-\varepsilon \mu}{(1+\varepsilon)(1-\mu)} \frac{1-\mu+\varepsilon \mu}{(1-\varepsilon)(1-\mu)}\right] .
$$

Since $\frac{1-\mu-\varepsilon \mu}{(1+\varepsilon)(1-\mu)} \frac{1-\mu+\varepsilon \mu}{(1-\varepsilon)(1-\mu)}=\frac{(1-\mu)^{2}-\varepsilon^{2} \mu^{2}}{(1-\mu)^{2}-\varepsilon^{2}(1-\mu)^{2}}>1$ for $0<\mu<\frac{1}{2}$, we have $\frac{\partial \mathscr{M}_{\mathrm{B}}(\mu+\varepsilon \mu, \mu)}{\partial \varepsilon}-\frac{\partial \mathscr{M}_{\mathrm{B}}(\mu-\varepsilon \mu, \mu)}{\partial \varepsilon}>0$ for $0<\mu<\frac{1}{2}$. Noting that $\mathscr{M}_{\mathrm{B}}(\mu+\varepsilon \mu, \mu)-\mathscr{M}_{\mathrm{B}}(\mu-\varepsilon \mu, \mu)=0$ for $\varepsilon=0$, we have $\mathscr{M}_{\mathrm{B}}(\mu+$ $\varepsilon \mu, \mu)-\mathscr{M}_{\mathrm{B}}(\mu-\varepsilon \mu, \mu)>0$ for $0<\mu<\frac{1}{2}$. This completes the proof of the lemma.

Lemma $14 \operatorname{Pr}\left\{\widehat{\boldsymbol{p}} \geq\left(1+\varepsilon_{r}\right) p\right\}<\frac{\delta}{2}$ for any $p \in\left(p^{\star}, 1\right)$. 
Proof. To prove the lemma, we shall consider the following three cases:

Case (i): $\left(1+\varepsilon_{r}\right) p>1$;

Case (ii): $\frac{\gamma}{n} \leq\left(1+\varepsilon_{r}\right) p \leq 1$;

Case (iii): $\left(1+\varepsilon_{r}\right) p<\frac{\gamma}{n}$.

For Case (i), it is obvious that $\operatorname{Pr}\left\{\widehat{\boldsymbol{p}} \geq\left(1+\varepsilon_{r}\right) p\right\}=0<\frac{\delta}{2}$.

For Case (ii), applying Theorem 1 with $z=\left(1+\varepsilon_{r}\right) p \geq \frac{\gamma}{n}$, we have

$$
\begin{aligned}
\operatorname{Pr}\left\{\widehat{\boldsymbol{p}} \geq\left(1+\varepsilon_{r}\right) p\right\} & =\operatorname{Pr}\left\{\sum_{i=1}^{\lfloor\gamma / z\rfloor} X_{i} \geq \gamma\right\} \\
& \leq \exp \left(\lfloor\gamma / z\rfloor \mathscr{M}_{\mathrm{B}}\left(\frac{\gamma}{\lfloor\gamma / z\rfloor}, p\right)\right) \\
& =\exp \left(\gamma \mathscr{M}_{\mathrm{I}}\left(\frac{\gamma}{\lfloor\gamma / z\rfloor}, p\right)\right) \\
& \leq \exp \left(\gamma \mathscr{M}_{\mathrm{I}}(z, p)\right) \\
& <\exp \left(\gamma \mathscr{M}_{\mathrm{I}}\left(p^{\star}+\varepsilon_{r} p^{\star}, p^{\star}\right)\right) \\
& <\frac{\delta}{2}
\end{aligned}
$$

where (12) follows from Lemma 5 , (13) is due to the fact that $\mathscr{M}_{\mathrm{I}}(z, p)$ is monotonically decreasing with respect to $z \in(p, 1)$, (14) follows from Lemma 6, and (15) follows from the assumption about $\gamma$

For Case (iii), applying Theorem 1 with $z=\left(1+\varepsilon_{r}\right) p<\frac{\gamma}{n}$, we have

$$
\begin{aligned}
\operatorname{Pr}\left\{\widehat{\boldsymbol{p}} \geq\left(1+\varepsilon_{r}\right) p\right\} & =\operatorname{Pr}\left\{\sum_{i=1}^{n} X_{i} \geq n z\right\} \\
& \leq \exp \left(n \mathscr{M}_{\mathrm{B}}\left(p+\varepsilon_{r} p, p\right)\right) \\
& <\exp \left(n \mathscr{M}_{\mathrm{B}}\left(p^{\star}+\varepsilon_{r} p^{\star}, p^{\star}\right)\right) \\
& =\exp \left(n \mathscr{M}_{\mathrm{B}}\left(p^{\star}+\varepsilon_{a}, p^{\star}\right)\right) \\
& <\frac{\delta}{2}
\end{aligned}
$$

where (16) follows from Lemma 5, (17) follows from the first statement of Lemma 12, and (18) follows from the assumption about $n$.

In summary, we have shown $\operatorname{Pr}\left\{\widehat{\boldsymbol{p}} \geq\left(1+\varepsilon_{r}\right) p\right\}<\frac{\delta}{2}$ for all cases. This completes the proof of the lemma.

Lemma $15 \operatorname{Pr}\left\{\widehat{\boldsymbol{p}} \leq\left(1-\varepsilon_{r}\right) p\right\}<\frac{\delta}{2}$ for any $p \in\left(p^{\star}, 1\right)$. 
Proof. To prove the lemma, we shall consider the following two cases:

Case (i): $\left(1-\varepsilon_{r}\right) p \geq \frac{\gamma}{n}$;

Case (ii): $\left(1-\varepsilon_{r}\right) p<\frac{\gamma}{n}$.

For Case (i), applying Theorem 1 with $z=\left(1-\varepsilon_{r}\right) p \geq \frac{\gamma}{n}$, we have

$$
\begin{aligned}
\operatorname{Pr}\left\{\widehat{\boldsymbol{p}} \leq\left(1-\varepsilon_{r}\right) p\right\} & =\operatorname{Pr}\left\{\sum_{i=1}^{\lceil\gamma / z\rceil-1} X_{i}<\gamma\right\}=\operatorname{Pr}\left\{\sum_{i=1}^{\lceil\gamma / z\rceil-1} X_{i} \leq \gamma-1\right\} \leq \operatorname{Pr}\left\{\sum_{i=1}^{\lceil\gamma / z\rceil} X_{i} \leq \gamma\right\} \\
& \leq \exp \left(\lceil\gamma / z\rceil \mathscr{M}_{\mathrm{B}}\left(\frac{\gamma}{\lceil\gamma / z\rceil}, p\right)\right) \\
& =\exp \left(\gamma \mathscr{M}_{\mathrm{I}}\left(\frac{\gamma}{\lceil\gamma / z\rceil}, p\right)\right) \\
& \leq \exp \left(\gamma \mathscr{M}_{\mathrm{I}}(z, p)\right) \\
& <\exp \left(\gamma \mathscr{M}_{\mathrm{I}}\left(p^{\star}-\varepsilon_{r} p^{\star}, p^{\star}\right)\right) \\
& <\exp \left(\gamma \mathscr{M}_{\mathrm{I}}\left(p^{\star}+\varepsilon_{r} p^{\star}, p^{\star}\right)\right) \\
& <\frac{\delta}{2}
\end{aligned}
$$

where (19) follows from Lemma 5, (20) is due to the fact that $\mathscr{M}_{\mathrm{I}}(z, p)$ is monotonically increasing with respect to $z \in(0, p)$, (21) follows from Lemma 6, and (22) follows from Lemma 7 ,

For Case (ii), applying Theorem 1 with $z=\left(1-\varepsilon_{r}\right) p<\frac{\gamma}{n}$, we have

$$
\begin{aligned}
\operatorname{Pr}\left\{\widehat{\boldsymbol{p}} \leq\left(1-\varepsilon_{r}\right) p\right\} & \leq \exp \left(n \mathscr{M}_{\mathrm{B}}\left(p-\varepsilon_{r} p, p\right)\right) \\
& <\exp \left(n \mathscr{M}_{\mathrm{B}}\left(p^{\star}-\varepsilon_{r} p^{\star}, p^{\star}\right)\right) \\
& <\exp \left(n \mathscr{M}_{\mathrm{B}}\left(p^{\star}+\varepsilon_{r} p^{\star}, p^{\star}\right)\right) \\
& =\exp \left(n \mathscr{M}_{\mathrm{B}}\left(p^{\star}+\varepsilon_{a}, p^{\star}\right)\right)<\frac{\delta}{2}
\end{aligned}
$$

where (23) follows from Lemma 5, (24) follows from the second statement of Lemma 12, and (25) follows from Lemma 13.

In summary, we have shown $\operatorname{Pr}\left\{\widehat{\boldsymbol{p}} \leq\left(1-\varepsilon_{r}\right) p\right\}<\frac{\delta}{2}$ for both cases. The lemma is thus proved.

Lemma $16 \operatorname{Pr}\left\{\widehat{\boldsymbol{p}} \geq p+\varepsilon_{a}\right\}<\frac{\delta}{2}$ for any $p \in\left(0, p^{\star}\right]$.

Proof. To prove the lemma, we shall consider the following two cases:

Case (i): $p+\varepsilon_{a} \geq \frac{\gamma}{n}$;

Case (ii): $p+\varepsilon_{a}<\frac{\gamma}{n}$. 
For Case (i), applying Theorem 1 with $z=p+\varepsilon_{a} \geq \frac{\gamma}{n}$, we have

$$
\begin{aligned}
\operatorname{Pr}\left\{\widehat{\boldsymbol{p}} \geq p+\varepsilon_{a}\right\} & =\operatorname{Pr}\left\{\sum_{i=1}^{\lfloor\gamma / z\rfloor} X_{i} \geq \gamma\right\} \leq \exp \left(\lfloor\gamma / z\rfloor \mathscr{M}_{\mathrm{B}}\left(\frac{\gamma}{\lfloor\gamma / z\rfloor}, p\right)\right) \\
& =\exp \left(\gamma \mathscr{M}_{\mathrm{I}}\left(\frac{\gamma}{\lfloor\gamma / z\rfloor}, p\right)\right) \leq \exp \left(\gamma \mathscr{M}_{\mathrm{I}}(z, p)\right) \\
& <\exp \left(\gamma \mathscr{M}_{\mathrm{I}}\left(p^{\star}+\varepsilon_{r} p^{\star}, p^{\star}\right)\right) \\
& <\frac{\delta}{2}
\end{aligned}
$$

where (26) follows from Lemma 8 .

For Case (ii), applying Theorem 1 with $z=p+\varepsilon_{a}<\frac{\gamma}{n}$, we have

$$
\begin{aligned}
\operatorname{Pr}\left\{\widehat{\boldsymbol{p}} \geq p+\varepsilon_{a}\right\} & =\operatorname{Pr}\left\{\sum_{i=1}^{n} X_{i} \geq n z\right\} \\
& \leq \exp \left(n \mathscr{M}_{\mathrm{B}}\left(p+\varepsilon_{a}, p\right)\right) \\
& \leq \exp \left(n \mathscr{M}_{\mathrm{B}}\left(p^{\star}+\varepsilon_{a}, p^{\star}\right)\right) \\
& <\frac{\delta}{2}
\end{aligned}
$$

where (27) follows from Lemma 15, (28) follows from the first statement of Lemma 10.

In summary, we have shown $\operatorname{Pr}\left\{\widehat{\boldsymbol{p}} \geq p+\varepsilon_{a}\right\}<\frac{\delta}{2}$ for both cases. The lemma is thus proved.

Lemma $17 \operatorname{Pr}\left\{\widehat{\boldsymbol{p}} \leq p-\varepsilon_{a}\right\}<\frac{\delta}{2}$ for any $p \in\left(0, p^{\star}\right]$.

Proof. To prove the lemma, we shall consider the following three cases:

Case (i): $p<\varepsilon_{a}$;

Case (ii): $p-\varepsilon_{a} \geq \frac{\gamma}{n}$;

Case (iii): $0 \leq p-\varepsilon_{a}<\frac{\gamma}{n}$.

For Case (i), it is obvious that $\operatorname{Pr}\left\{\widehat{\boldsymbol{p}} \leq p-\varepsilon_{a}\right\}=0<\frac{\delta}{2}$.

For Case (ii), applying Theorem 1 with $z=p-\varepsilon_{a} \geq \frac{\gamma}{n}$, we have

$$
\begin{aligned}
\operatorname{Pr}\left\{\widehat{\boldsymbol{p}} \leq p-\varepsilon_{a}\right\} & =\operatorname{Pr}\left\{\sum_{i=1}^{\lceil\gamma / z\rceil-1} X_{i}<\gamma\right\} \leq \operatorname{Pr}\left\{\sum_{i=1}^{\lceil\gamma / z\rceil} X_{i} \leq \gamma\right\} \leq \exp \left(\lceil\gamma / z\rceil \mathscr{M}_{\mathrm{B}}\left(\frac{\gamma}{\lceil\gamma / z\rceil}, p\right)\right) \\
& =\exp \left(\gamma \mathscr{M}_{\mathrm{I}}\left(\frac{\gamma}{\lceil\gamma / z\rceil}, p\right)\right) \leq \exp \left(\gamma \mathscr{M}_{\mathrm{I}}(z, p)\right)=\exp \left(\gamma \mathscr{M}_{\mathrm{I}}\left(p-\varepsilon_{a}, p\right)\right) \\
& \leq \exp \left(\gamma \mathscr{M}_{\mathrm{I}}\left(p^{\star}-\varepsilon_{a}, p^{\star}\right)\right) \\
& \leq \exp \left(\gamma \mathscr{M}_{\mathrm{I}}\left(p^{\star}+\varepsilon_{a}, p^{\star}\right)\right) \\
& <\frac{\delta}{2}
\end{aligned}
$$


where (29) follows from the second statement of Lemma 8, and (30) follows from Lemma 9 ,

For Case (iii), applying Theorem 1 with $z=p-\varepsilon_{a}<\frac{\gamma}{n}$, we have

$$
\begin{aligned}
\operatorname{Pr}\left\{\widehat{\boldsymbol{p}} \leq p-\varepsilon_{a}\right\} & =\operatorname{Pr}\left\{\sum_{i=1}^{n} X_{i} \leq n z\right\} \\
& \leq \exp \left(n \mathscr{M}_{\mathrm{B}}\left(p-\varepsilon_{a}, p\right)\right) \\
& \leq \exp \left(n \mathscr{M}_{\mathrm{B}}\left(p^{\star}-\varepsilon_{a}, p^{\star}\right)\right) \\
& \leq \exp \left(n \mathscr{M}_{\mathrm{B}}\left(p^{\star}+\varepsilon_{a}, p^{\star}\right)\right) \\
& <\frac{\delta}{2}
\end{aligned}
$$

where (31) follows from Lemma 5, (32) follows from the second statement of Lemma 10, and (33) follows from Lemma 11]

In summary, we have shown $\operatorname{Pr}\left\{\widehat{\boldsymbol{p}} \leq p-\varepsilon_{a}\right\}<\frac{\delta}{2}$ for all cases. The lemma is thus proved.

Now we are in a position to prove Theorem 3. To show $\operatorname{Pr}\left\{|\widehat{\boldsymbol{p}}-p|<\varepsilon_{a}\right.$ or $\left.|\widehat{\boldsymbol{p}}-p|<\varepsilon_{r} p\right\}>$ $1-\delta$, it suffices to show $\operatorname{Pr}\left\{|\widehat{\boldsymbol{p}}-p| \geq \varepsilon_{a},|\widehat{\boldsymbol{p}}-p| \geq \varepsilon_{r} p\right\}<\delta$ for $0<p<1$.

For $p \in\left(p^{\star}, 1\right)$, we have

$$
\begin{aligned}
\operatorname{Pr}\left\{|\widehat{\boldsymbol{p}}-p| \geq \varepsilon_{a},|\widehat{\boldsymbol{p}}-p| \geq \varepsilon_{r} p\right\} & =\operatorname{Pr}\left\{|\widehat{\boldsymbol{p}}-p| \geq \varepsilon_{r} p\right\}=\operatorname{Pr}\left\{\widehat{\boldsymbol{p}} \geq\left(1+\varepsilon_{r}\right) p\right\}+\operatorname{Pr}\left\{\widehat{\boldsymbol{p}} \leq\left(1-\varepsilon_{r}\right) p\right\} \\
& <\frac{\delta}{2}+\frac{\delta}{2} \\
& =\delta
\end{aligned}
$$

where (34) follows from Lemmas 14 and 15, Similarly, for $p \in\left(0, p^{\star}\right]$, we have

$$
\begin{aligned}
\operatorname{Pr}\left\{|\widehat{\boldsymbol{p}}-p| \geq \varepsilon_{a},|\widehat{\boldsymbol{p}}-p| \geq \varepsilon_{r} p\right\} & =\operatorname{Pr}\left\{|\widehat{\boldsymbol{p}}-p| \geq \varepsilon_{a}\right\}=\operatorname{Pr}\left\{\widehat{\boldsymbol{p}} \geq p+\varepsilon_{a}\right\}+\operatorname{Pr}\left\{\widehat{\boldsymbol{p}} \leq p-\varepsilon_{a}\right\} \\
& <\frac{\delta}{2}+\frac{\delta}{2} \\
& =\delta
\end{aligned}
$$

where (35) follows from Lemmas 16] and 17. This completes the proof of Theorem 3.

\section{Proof of Theorem 4}

Lemma 18 Let I denote the support of $\widehat{\boldsymbol{p}}$. Suppose the intersection between open interval $\left(p^{\prime}, p^{\prime \prime}\right)$ and set $I_{\mathscr{L}}$ is empty. Then, $\{\vartheta \in I: p \leq \mathscr{L}(\vartheta)\}$ is fixed with respect to $p \in\left(p^{\prime}, p^{\prime \prime}\right)$.

Proof. Let $p^{*}$ and $p^{\diamond}$ be two distinct real numbers included in interval $\left(p^{\prime}, p^{\prime \prime}\right)$. To show the lemma, it suffices to show that $\left\{\vartheta \in I: p^{*} \leq \mathscr{L}(\vartheta)\right\}=\left\{\vartheta \in I: p^{\diamond} \leq \mathscr{L}(\vartheta)\right\}$. First, we shall show that $\left\{\vartheta \in I: p^{*} \leq \mathscr{L}(\vartheta)\right\} \subseteq\left\{\vartheta \in I: p^{\diamond} \leq \mathscr{L}(\vartheta)\right\}$. To this end, we let $\varpi \in\left\{\vartheta \in I: p^{*} \leq \mathscr{L}(\vartheta)\right\}$ and proceed to show $\varpi \in\left\{\vartheta \in I: p^{\diamond} \leq \mathscr{L}(\vartheta)\right\}$. Since $\varpi \in I$ and $p^{*} \leq \mathscr{L}(\varpi)$, it must be true 
that $\varpi \in I$ and $p^{\diamond} \leq \mathscr{L}(\varpi)$. If this is not the case, then we have $p^{\prime \prime}>p^{\diamond}>\mathscr{L}(\varpi) \geq p^{*}>p^{\prime}$. Consequently, $\mathscr{L}(\varpi)$ is included by both the interval $\left(p^{\prime}, p^{\prime \prime}\right)$ and the set $I \mathscr{L}$. This contradicts the assumption of the lemma. Hence, we have shown $\varpi \in\left\{\vartheta \in I: p^{\diamond} \leq \mathscr{L}(\vartheta)\right\}$ and accordingly $\left\{\vartheta \in I: p^{*} \leq \mathscr{L}(\vartheta)\right\} \subseteq\left\{\vartheta \in I: p^{\diamond} \leq \mathscr{L}(\vartheta)\right\}$. Second, by a similar argument, we can show $\left\{\vartheta \in I: p^{\diamond} \leq \mathscr{L}(\vartheta)\right\} \subseteq\left\{\vartheta \in I: p^{*} \leq \mathscr{L}(\vartheta)\right\}$. It follows that $\left\{\vartheta \in I: p^{*} \leq \mathscr{L}(\vartheta)\right\}=\left\{\vartheta \in I: p^{\diamond} \leq\right.$ $\mathscr{L}(\vartheta)\}$. Finally, the proof of the lemma is completed by noting that the above argument holds for arbitrary $p^{*}$ and $p^{\diamond}$ included in the open interval $\left(p^{\prime}, p^{\prime \prime}\right)$.

By virtue of Theorem 1, we can show the following lemma.

Lemma $19 \operatorname{Pr}\{\widehat{\boldsymbol{p}} \leq z \mid p\}$ is monotonically decreasing with respect to $p$. Similarly, $\operatorname{Pr}\{\widehat{\boldsymbol{p}} \geq z \mid p\}$ is monotonically increasing with respect to $p$.

Lemma 20 Let $p^{\prime}<p^{\prime \prime}$ be two consecutive distinct elements of $I_{\mathscr{L}} \cap[a, b] \cup\{a, b\}$. Then,

$$
\begin{aligned}
& \lim _{\epsilon \downarrow 0} \operatorname{Pr}\left\{p^{\prime}+\epsilon \leq \mathscr{L}(\widehat{\boldsymbol{p}}) \mid p^{\prime}+\epsilon\right\}=\operatorname{Pr}\left\{p^{\prime}<\mathscr{L}(\widehat{\boldsymbol{p}}) \mid p^{\prime}\right\}, \\
& \lim _{\epsilon \downarrow 0} \operatorname{Pr}\left\{p^{\prime \prime}-\epsilon \leq \mathscr{L}(\widehat{\boldsymbol{p}}) \mid p^{\prime \prime}-\epsilon\right\}=\operatorname{Pr}\left\{p^{\prime \prime} \leq \mathscr{L}(\widehat{\boldsymbol{p}}) \mid p^{\prime \prime}\right\} .
\end{aligned}
$$

Moreover, $\operatorname{Pr}\{p \leq \mathscr{L}(\widehat{\boldsymbol{p}}) \mid p\}$ is monotone with respect to $p \in\left(p^{\prime}, p^{\prime \prime}\right)$.

Proof. First, we shall show that $\lim _{\epsilon \downarrow 0} \operatorname{Pr}\left\{p^{\prime}+\epsilon \leq \mathscr{L}(\widehat{\boldsymbol{p}}) \mid p^{\prime}+\epsilon\right\}=\operatorname{Pr}\left\{p^{\prime}<\mathscr{L}(\widehat{\boldsymbol{p}}) \mid p^{\prime}\right\}$. Let $m^{+}(\epsilon)$ be the number of elements of $\left\{\vartheta \in I: p^{\prime}<\mathscr{L}(\vartheta)<p^{\prime}+\epsilon\right\}$, where $I$ denotes the support of $\widehat{\boldsymbol{p}}$ as in Lemma 18. We claim that $\lim _{\epsilon \downarrow 0} m^{+}(\epsilon)=0$. It suffices to consider two cases as follows.

In the case of $\left\{\vartheta \in I: p^{\prime}<\mathscr{L}(\vartheta)\right\}=\emptyset$, we have $m^{+}(\epsilon)=0$ for any $\epsilon>0$. In the case of $\{\vartheta \in I$ : $\left.p^{\prime}<\mathscr{L}(\vartheta)\right\} \neq \emptyset$, we have $m^{+}(\epsilon)=0$ for $0<\epsilon \leq \epsilon^{*}$, where $\epsilon^{*}=\min \left\{\mathscr{L}(\vartheta)-p^{\prime}: p^{\prime}<\mathscr{L}(\vartheta), \vartheta \in I\right\}$ is positive because of the assumption that $I_{\mathscr{L}}$ has no closure points in $[a, b]$. Hence, in both cases, $\lim _{\epsilon \downarrow 0} m^{+}(\epsilon)=0$. This establishes the claim.

Noting that $\operatorname{Pr}\left\{p^{\prime}<\mathscr{L}(\widehat{\boldsymbol{p}})<p^{\prime}+\epsilon \mid p^{\prime}+\epsilon\right\} \leq m^{+}(\epsilon)$ as a consequence of $\operatorname{Pr}\left\{\widehat{\boldsymbol{p}}=\vartheta \mid p^{\prime}+\epsilon\right\} \leq 1$ for any $\vartheta \in I$, we have that $\limsup _{\epsilon \downarrow 0} \operatorname{Pr}\left\{p^{\prime}<\mathscr{L}(\widehat{\boldsymbol{p}})<p^{\prime}+\epsilon \mid p^{\prime}+\epsilon\right\} \leq \lim _{\epsilon \downarrow 0} m^{+}(\epsilon)=0$, which implies that $\lim _{\epsilon \downarrow 0} \operatorname{Pr}\left\{p^{\prime}<\mathscr{L}(\widehat{\boldsymbol{p}})<p^{\prime}+\epsilon \mid p^{\prime}+\epsilon\right\}=0$.

Since $\left\{p^{\prime}+\epsilon \leq \mathscr{L}(\widehat{\boldsymbol{p}})\right\} \cap\left\{p^{\prime}<\mathscr{L}(\widehat{\boldsymbol{p}})<p^{\prime}+\epsilon\right\}=\emptyset$ and $\left\{p^{\prime}<\mathscr{L}(\widehat{\boldsymbol{p}})\right\}=\left\{p^{\prime}+\epsilon \leq \mathscr{L}(\widehat{\boldsymbol{p}})\right\} \cup\left\{p^{\prime}<\right.$ $\left.\mathscr{L}(\widehat{\boldsymbol{p}})<p^{\prime}+\epsilon\right\}$, we have $\operatorname{Pr}\left\{p^{\prime}<\mathscr{L}(\widehat{\boldsymbol{p}}) \mid p^{\prime}+\epsilon\right\}=\operatorname{Pr}\left\{p^{\prime}+\epsilon \leq \mathscr{L}(\widehat{\boldsymbol{p}}) \mid p^{\prime}+\epsilon\right\}+\operatorname{Pr}\left\{p^{\prime}<\mathscr{L}(\widehat{\boldsymbol{p}})<\right.$ $\left.p^{\prime}+\epsilon \mid p^{\prime}+\epsilon\right\}$. Observing that $\operatorname{Pr}\left\{p^{\prime}<\mathscr{L}(\widehat{\boldsymbol{p}}) \mid p^{\prime}+\epsilon\right\}$ is continuous with respect to $\epsilon \in\left(0,1-p^{\prime}\right)$, we have $\lim _{\epsilon \downarrow 0} \operatorname{Pr}\left\{p^{\prime}<\mathscr{L}(\widehat{\boldsymbol{p}}) \mid p^{\prime}+\epsilon\right\}=\operatorname{Pr}\left\{p^{\prime}<\mathscr{L}(\widehat{\boldsymbol{p}}) \mid p^{\prime}\right\}$. It follows that

$$
\begin{aligned}
\lim _{\epsilon \downarrow 0} \operatorname{Pr}\left\{p^{\prime}+\epsilon \leq \mathscr{L}(\widehat{\boldsymbol{p}}) \mid p^{\prime}+\epsilon\right\} & =\lim _{\epsilon \downarrow 0} \operatorname{Pr}\left\{p^{\prime}<\mathscr{L}(\widehat{\boldsymbol{p}}) \mid p^{\prime}+\epsilon\right\}-\lim _{\epsilon \downarrow 0} \operatorname{Pr}\left\{p^{\prime}<\mathscr{L}(\widehat{\boldsymbol{p}})<p^{\prime}+\epsilon \mid p^{\prime}+\epsilon\right\} \\
& =\lim _{\epsilon \downarrow 0} \operatorname{Pr}\left\{p^{\prime}<\mathscr{L}(\widehat{\boldsymbol{p}}) \mid p^{\prime}+\epsilon\right\}=\operatorname{Pr}\left\{p^{\prime}<\mathscr{L}(\widehat{\boldsymbol{p}}) \mid p^{\prime}\right\} .
\end{aligned}
$$


Next, we shall show that $\lim _{\epsilon \downarrow 0} \operatorname{Pr}\left\{p^{\prime \prime}-\epsilon \leq \mathscr{L}(\widehat{\boldsymbol{p}}) \mid p^{\prime \prime}-\epsilon\right\}=\operatorname{Pr}\left\{p^{\prime \prime} \leq \mathscr{L}(\widehat{\boldsymbol{p}}) \mid p^{\prime \prime}\right\}$. Let $m^{-}(\epsilon)$ be the number of elements of $\left\{\vartheta \in I: p^{\prime \prime}-\epsilon \leq \mathscr{L}(\vartheta)<p^{\prime \prime}\right\}$. Then, we can show $\lim _{\epsilon \downarrow 0} m^{-}(\epsilon)=0$ by considering two cases as follows.

In the case of $\left\{\vartheta \in I: \mathscr{L}(\vartheta)<p^{\prime \prime}\right\}=\emptyset$, we have $m^{-}(\epsilon)=0$ for any $\epsilon>0$. In the case of $\left\{\vartheta \in I: \mathscr{L}(\vartheta)<p^{\prime \prime}\right\} \neq \emptyset$, we have $m^{-}(\epsilon)=0$ for $0<\epsilon<\epsilon^{\star}$, where $\epsilon^{\star}=\min \left\{p^{\prime \prime}-\mathscr{L}(\vartheta): \vartheta \in\right.$ $\left.I, \mathscr{L}(\vartheta)<p^{\prime \prime}\right\}$ is positive because of the assumption that $I_{\mathscr{U}}$ has no closure points in $[a, b]$. Hence, in both cases, $\lim _{\epsilon \downarrow 0} m^{-}(\epsilon)=0$. It follows that $\lim _{\sup _{\epsilon \downarrow 0}} \operatorname{Pr}\left\{p^{\prime \prime}-\epsilon \leq \mathscr{L}(\widehat{\boldsymbol{p}})<p^{\prime \prime} \mid p^{\prime \prime}-\epsilon\right\} \leq$ $\lim _{\epsilon \downarrow 0} m^{-}(\epsilon)=0$ and consequently $\lim _{\epsilon \downarrow 0} \operatorname{Pr}\left\{p^{\prime \prime}-\epsilon \leq \mathscr{L}(\widehat{\boldsymbol{p}})<p^{\prime \prime} \mid p^{\prime \prime}-\epsilon\right\}=0$.

Since $\left\{p^{\prime \prime}-\epsilon \leq \mathscr{L}(\widehat{\boldsymbol{p}})\right\}=\left\{p^{\prime \prime} \leq \mathscr{L}(\widehat{\boldsymbol{p}})\right\} \cup\left\{p^{\prime \prime}-\epsilon \leq \mathscr{L}(\widehat{\boldsymbol{p}})<p^{\prime \prime}\right\}$ and $\left\{p^{\prime \prime} \leq \mathscr{L}(\widehat{\boldsymbol{p}})\right\} \cap\left\{p^{\prime \prime}-\epsilon \leq\right.$ $\left.\mathscr{L}(\widehat{\boldsymbol{p}})<p^{\prime \prime}\right\}=\emptyset$, we have $\operatorname{Pr}\left\{p^{\prime \prime}-\epsilon \leq \mathscr{L}(\widehat{\boldsymbol{p}}) \mid p^{\prime \prime}-\epsilon\right\}=\operatorname{Pr}\left\{p^{\prime \prime} \leq \mathscr{L}(\widehat{\boldsymbol{p}}) \mid p^{\prime \prime}-\epsilon\right\}+\operatorname{Pr}\left\{p^{\prime \prime}-\epsilon \leq\right.$ $\left.\mathscr{L}(\widehat{\boldsymbol{p}})<p^{\prime \prime} \mid p^{\prime \prime}-\epsilon\right\}$.

Observing that $\operatorname{Pr}\left\{p^{\prime \prime} \leq \mathscr{L}(\widehat{\boldsymbol{p}}) \mid p^{\prime \prime}-\epsilon\right\}$ is continuous with respect to $\epsilon \in\left(0, p^{\prime \prime}\right)$, we have $\lim _{\epsilon \downarrow 0} \operatorname{Pr}\left\{p^{\prime \prime} \leq \mathscr{L}(\widehat{\boldsymbol{p}}) \mid p^{\prime \prime}-\epsilon\right\}=\operatorname{Pr}\left\{p^{\prime \prime} \leq \mathscr{L}(\widehat{\boldsymbol{p}}) \mid p^{\prime \prime}\right\}$. It follows that $\lim _{\epsilon \downarrow 0} \operatorname{Pr}\left\{p^{\prime \prime}-\epsilon \leq \mathscr{L}(\widehat{\boldsymbol{p}}) \mid\right.$ $\left.p^{\prime \prime}-\epsilon\right\}=\lim _{\epsilon \downarrow 0}\left\{p^{\prime \prime} \leq \mathscr{L}(\widehat{\boldsymbol{p}}) \mid p^{\prime \prime}\right\}$.

Now we turn to show that $\operatorname{Pr}\{p \leq \mathscr{L}(\widehat{\boldsymbol{p}}) \mid p\}$ is monotone with respect to $p \in\left(p^{\prime}, p^{\prime \prime}\right)$. Without loss of generality, we assume that $\mathscr{L}($.$) is monotonically increasing. Since p^{\prime}<p^{\prime \prime}$ are two consecutive distinct elements of $I_{\mathscr{L}} \cap[a, b] \cup\{a, b\}$, we have that the intersection between open interval $\left(p^{\prime}, p^{\prime \prime}\right)$ and set $I_{\mathscr{L}}$ is empty. As a result of Lemma 18, we can write $\operatorname{Pr}\{p \leq \mathscr{L}(\widehat{\boldsymbol{p}}) \mid$ $p\}=\operatorname{Pr}\{\widehat{\boldsymbol{p}} \geq \vartheta \mid p\}$, where $\vartheta \in[0,1]$ is a constant independent of $p \in\left(p^{\prime}, p^{\prime \prime}\right)$. By Lemma 19, we have that $\operatorname{Pr}\{\widehat{\boldsymbol{p}} \geq \vartheta \mid p\}$ is monotonically increasing with respect to $p \in\left(p^{\prime}, p^{\prime \prime}\right)$. This proves the monotonicity of $\operatorname{Pr}\{p \leq \mathscr{L}(\widehat{\boldsymbol{p}}) \mid p\}$ with respect to $p \in\left(p^{\prime}, p^{\prime \prime}\right)$. The proof of the lemma is thus completed.

By a similar method as that of Lemma 20, we can show the following lemma.

Lemma 21 Let $p^{\prime}<p^{\prime \prime}$ be two consecutive distinct elements of $I_{\mathscr{U}} \cap[a, b] \cup\{a, b\}$. Then,

$$
\begin{aligned}
& \lim _{\epsilon \downarrow 0} \operatorname{Pr}\left\{p^{\prime}+\epsilon \geq \mathscr{U}(\widehat{\boldsymbol{p}}) \mid p^{\prime}+\epsilon\right\}=\operatorname{Pr}\left\{p^{\prime} \geq \mathscr{U}(\widehat{\boldsymbol{p}}) \mid p^{\prime}\right\}, \\
& \lim _{\epsilon \downarrow 0} \operatorname{Pr}\left\{p^{\prime \prime}-\epsilon \geq \mathscr{U}(\widehat{\boldsymbol{p}}) \mid p^{\prime \prime}-\epsilon\right\}=\operatorname{Pr}\left\{p^{\prime \prime}>\mathscr{U}(\widehat{\boldsymbol{p}}) \mid p^{\prime \prime}\right\} .
\end{aligned}
$$

Moreover, $\operatorname{Pr}\{p \geq \mathscr{U}(\widehat{\boldsymbol{p}}) \mid p\}$ is monotone with respect to $p \in\left(p^{\prime}, p^{\prime \prime}\right)$.

Now we are in a position to prove Theorem 4, Let $C(p)=\operatorname{Pr}\{p \leq \mathscr{L}(\widehat{\boldsymbol{p}}) \mid p\}$. By Lemma 20. $C(p)$ is a monotone function of $p \in\left(p^{\prime}, p^{\prime \prime}\right)$, which implies that $C(p) \leq \max \left\{C\left(p^{\prime}+\epsilon\right), C\left(p^{\prime \prime}-\epsilon\right)\right\}$ for any $p \in\left(p^{\prime}, p^{\prime \prime}\right)$ and any positive $\epsilon$ less than $\min \left\{p-p^{\prime}, p^{\prime \prime}-p\right\}$. Consequently,

$C(p) \leq \lim _{\epsilon \downarrow 0} \max \left\{C\left(p^{\prime}+\epsilon\right), C\left(p^{\prime \prime}-\epsilon\right)\right\}=\max \left\{\lim _{\epsilon \downarrow 0} C\left(p^{\prime}+\epsilon\right), \lim _{\epsilon \downarrow 0} C\left(p^{\prime \prime}-\epsilon\right)\right\} \leq \max \left\{C\left(p^{\prime}\right), C\left(p^{\prime \prime}\right)\right\}$

for any $p \in\left(p^{\prime}, p^{\prime \prime}\right)$. Since the argument holds for arbitrary consecutive distinct elements of $\{\mathscr{L}(\widehat{p}) \in(a, b) \mid \widehat{p} \in I\} \cup\{a, b\}$, we have established the statement regarding the maximum of 
$\operatorname{Pr}\{p \leq \mathscr{L}(\widehat{\boldsymbol{p}}) \mid p\}$ with respect to $p \in(a, b)$. By a similar method, we can prove the statement regarding the maximum of $\operatorname{Pr}\{p \geq \mathscr{U}(\widehat{\boldsymbol{p}}) \mid p\}$ with respect to $p \in(a, b)$. This concludes the proof of Theorem 4 .

\section{E Proof of Theorem 6}

The theorem can be established by showing the following lemmas.

Lemma $22 \operatorname{Pr}\{p \geq \overline{\boldsymbol{p}}\} \leq \frac{\delta}{2}$.

Proof. By Theorem 1,

$$
\operatorname{Pr}\{\widehat{\boldsymbol{p}} \leq z\}= \begin{cases}\operatorname{Pr}\left\{\sum_{i=1}^{\lceil\gamma / z]-1} X_{i}<\gamma\right\} & \text { for } \gamma \leq n z, \\ \operatorname{Pr}\left\{\sum_{i=1}^{n} X_{i} \leq n z\right\} & \text { for } \gamma>n z .\end{cases}
$$

Since $X_{i}$ must be either 0 or 1 and $\gamma$ is an integer, we have $\operatorname{Pr}\left\{\sum_{i=1}^{\lceil\gamma / z\rceil-1} X_{i}<\gamma\right\} \leq \operatorname{Pr}\left\{\sum_{i=1}^{\lceil\gamma / z\rceil} X_{i} \leq\right.$ $\gamma\}$. Hence,

$$
\operatorname{Pr}\{\widehat{\boldsymbol{p}} \leq z\} \leq \begin{cases}\operatorname{Pr}\left\{\sum_{i=1}^{\lceil\gamma / z\rceil} X_{i} \leq \gamma\right\} & \text { for } \gamma \leq n z, \\ \operatorname{Pr}\left\{\sum_{i=1}^{n} X_{i} \leq n z\right\} & \text { for } \gamma>n z .\end{cases}
$$

Since $X_{1}, X_{2}, \cdots$ are i.i.d. Bernoulli random variables, we have $\operatorname{Pr}\{\widehat{\boldsymbol{p}} \leq z\} \leq \mathscr{G}(z, p)$, where

$$
\mathscr{G}(z, p)= \begin{cases}\sum_{i=0}^{\gamma}\left(\begin{array}{c}
\lceil\gamma / z\rceil \\
i
\end{array}\right) p^{i}(1-p)^{\lceil\gamma / z\rceil-i} & \text { for } \frac{\gamma}{n} \leq z \leq 1, \\
\sum_{i=0}^{\lfloor n z\rfloor}\left(\begin{array}{c}
n \\
i
\end{array}\right) p^{i}(1-p)^{n-i} & \text { for } 0 \leq z<\frac{\gamma}{n} .\end{cases}
$$

Let $z^{*} \in[0,1]$ be the largest number such that $\operatorname{Pr}\left\{\widehat{\boldsymbol{p}}<z^{*}\right\} \leq \frac{\delta}{2}$. Since $\widehat{\boldsymbol{p}}$ is a discrete random variable bounded in $[0,1]$, it must be true that $\operatorname{Pr}\left\{\widehat{\boldsymbol{p}} \leq z^{*}\right\}>\frac{\delta}{2}$. Observing that $\mathscr{G}(z, p)$ is monotonically decreasing with respect to $p \in(0,1)$, we have

$$
\{p \geq \overline{\boldsymbol{p}}\}=\{p \geq \overline{\boldsymbol{p}}, \mathbf{k}<\mathbf{n}\} \subseteq\left\{\mathscr{G}(\widehat{\boldsymbol{p}}, p) \leq \mathscr{G}(\widehat{\boldsymbol{p}}, \overline{\boldsymbol{p}})=\frac{\delta}{2}\right\} \subseteq\left\{\mathscr{G}(\widehat{\boldsymbol{p}}, p) \leq \frac{\delta}{2}\right\} .
$$

Noting that $\frac{\delta}{2}<\operatorname{Pr}\left\{\widehat{\boldsymbol{p}} \leq z^{*}\right\} \leq \mathscr{G}\left(z^{*}, p\right)$ and that $\mathscr{G}(z, p)$ is non-decreasing with respect to $z \in(0,1)$, we have $\{p \geq \overline{\boldsymbol{p}}\} \subseteq\left\{\mathscr{G}(\widehat{\boldsymbol{p}}, p) \leq \frac{\delta}{2}\right\} \subseteq\left\{\mathscr{G}(\widehat{\boldsymbol{p}}, p)<\mathscr{G}\left(z^{*}, p\right)\right\} \subseteq\left\{\widehat{\boldsymbol{p}}<z^{*}\right\}$. It follows that $\operatorname{Pr}\{p \geq \overline{\boldsymbol{p}}\} \leq \operatorname{Pr}\left\{\widehat{\boldsymbol{p}}<z^{*}\right\} \leq \frac{\delta}{2}$.

Lemma $23 \operatorname{Pr}\{p \leq \underline{\boldsymbol{p}}\} \leq \frac{\delta}{2}$. 
Proof. By Theorem 1,

$$
\operatorname{Pr}\{\widehat{\boldsymbol{p}} \geq z\}= \begin{cases}\operatorname{Pr}\left\{\sum_{i=1}^{\lfloor\gamma / z\rfloor} X_{i} \geq \gamma\right\} & \text { for } \gamma \leq n z \\ \operatorname{Pr}\left\{\sum_{i=1}^{n} X_{i} \geq n z\right\} & \text { for } \gamma>n z .\end{cases}
$$

Since $X_{1}, X_{2}, \cdots$ are i.i.d. Bernoulli random variables, we have $\operatorname{Pr}\{\widehat{\boldsymbol{p}} \geq z\}=\mathscr{H}(z, p)$ where

$$
\mathscr{H}(z, p)= \begin{cases}\sum_{i=\gamma}^{\lfloor\gamma / z\rfloor}\left(\begin{array}{c}
\lfloor\gamma / z\rfloor \\
i
\end{array}\right) p^{i}(1-p)^{\lfloor\gamma / z\rfloor-i} & \text { for } \frac{\gamma}{n} \leq z \leq 1, \\
\sum_{i=\lceil n z\rceil}^{n}\left(\begin{array}{c}
n \\
i
\end{array}\right) p^{i}(1-p)^{n-i} & \text { for } 0 \leq z<\frac{\gamma}{n}\end{cases}
$$

Let $z^{*} \in[0,1]$ be the smallest number such that $\operatorname{Pr}\left\{\widehat{\boldsymbol{p}}>z^{*}\right\} \leq \frac{\delta}{2}$. Since $\widehat{\boldsymbol{p}}$ is a discrete random variable bounded in $[0,1]$, it must be true that $\operatorname{Pr}\left\{\widehat{\boldsymbol{p}} \geq z^{*}\right\}>\frac{\delta}{2}$. Observing that $\mathscr{H}(z, p)$ is monotonically increasing with respect to $p \in(0,1)$, we have

$$
\{p \leq \underline{\boldsymbol{p}}\}=\{p \leq \underline{\boldsymbol{p}}, \mathbf{k}>0\} \subseteq\left\{\mathscr{H}(\widehat{\boldsymbol{p}}, p) \leq \mathscr{H}(\widehat{\boldsymbol{p}}, \underline{\boldsymbol{p}})=\frac{\delta}{2}\right\} \subseteq\left\{\mathscr{H}(\widehat{\boldsymbol{p}}, p) \leq \frac{\delta}{2}\right\} .
$$

Noting that $\frac{\delta}{2}<\operatorname{Pr}\left\{\widehat{\boldsymbol{p}} \geq z^{*}\right\}=\mathscr{H}\left(z^{*}, p\right)$ and that $\mathscr{H}(z, p)$ is non-increasing with respect to $z \in(0,1)$, we have $\{p \leq \underline{\boldsymbol{p}}\} \subseteq\left\{\mathscr{H}(\widehat{\boldsymbol{p}}, p) \leq \frac{\delta}{2}\right\} \subseteq\left\{\mathscr{H}(\widehat{\boldsymbol{p}}, p)<\mathscr{H}\left(z^{*}, p\right)\right\} \subseteq\left\{\widehat{\boldsymbol{p}}>z^{*}\right\}$. It follows that $\operatorname{Pr}\{p \leq \underline{\boldsymbol{p}}\} \leq \operatorname{Pr}\left\{\widehat{\boldsymbol{p}}>z^{*}\right\} \leq \frac{\delta}{2}$.

\section{F $\quad$ Proof of Theorem 7}

Theorem 7 can be shown by using the following result (a slight modification of Hoeffding's inequality [7]) and a similar argument as that of Theorem 3.

Lemma 24 Let $X_{1}, \cdots, X_{n}$ be random variables with joint distribution given by (5). Then, $\operatorname{Pr}\left\{\frac{\sum_{i=1}^{n} X_{i}}{n} \geq z\right\} \leq \exp \left(n \mathscr{M}_{\mathrm{B}}(z, p)\right)$ for $1 \geq z \geq p=\frac{M}{N}$. Similarly, $\operatorname{Pr}\left\{\frac{\sum_{i=1}^{n} X_{i}}{n} \leq z\right\} \leq \exp \left(n \mathscr{M}_{\mathrm{B}}(z, p)\right)$ for $0 \leq z \leq p$.

Proof. For $z=p$, we have $\operatorname{Pr}\left\{\frac{\sum_{i=1}^{n} X_{i}}{n} \geq z\right\} \leq \exp \left(n \mathscr{M}_{\mathrm{B}}(z, p)\right)=1$. For $p<z<1$, it was shown by Hoeffding in [7] that $\operatorname{Pr}\left\{\frac{\sum_{i=1}^{n} X_{i}}{n} \geq z\right\} \leq \exp \left(n \mathscr{M}_{\mathrm{B}}(z, p)\right)$. For $z=1, \operatorname{Pr}\left\{\frac{\sum_{i=1}^{n} X_{i}}{n} \geq z\right\}=\operatorname{Pr}\left\{X_{i}=\right.$ $1, i=1, \cdots, n\}=\left(\begin{array}{c}M \\ n\end{array}\right) /\left(\begin{array}{c}N \\ n\end{array}\right) \leq p^{n}=\exp \left(n \mathscr{M}_{\mathrm{B}}(1, p)\right)$.

For $z=0, \operatorname{Pr}\left\{\frac{\sum_{i=1}^{n} X_{i}}{n} \leq z\right\}=\operatorname{Pr}\left\{X_{i}=0, i=1, \cdots, n\right\}=\left(\begin{array}{c}N-M \\ n\end{array}\right) /\left(\begin{array}{c}N \\ n\end{array}\right) \leq(1-p)^{n}=\exp \left(n \mathscr{M}_{\mathrm{B}}(0, p)\right)$. For $0<z<p$, it was shown by Hoeffding in [7] that $\operatorname{Pr}\left\{\frac{\sum_{i=1}^{n} X_{i}}{n} \leq z\right\} \leq \exp \left(n \mathscr{M}_{\mathrm{B}}(z, p)\right)$. For $z=p$, we have $\operatorname{Pr}\left\{\frac{\sum_{i=1}^{n} X_{i}}{n} \leq z\right\} \leq \exp \left(n \mathscr{M}_{\mathrm{B}}(z, p)\right)=1$. 


\section{G Proof of Theorem 8}

By the same argument as that of Theorem 1, we can show the following lemma.

Lemma 25 For any $z>0$,

$$
\operatorname{Pr}\{\widehat{\boldsymbol{p}} \leq z\}=\left\{\begin{array}{ll}
\operatorname{Pr}\left\{\sum_{i=1}^{\lceil\gamma / z\rceil-1} X_{i}<\gamma\right\} & \text { for } \gamma \leq n z, \\
\operatorname{Pr}\left\{\sum_{i=1}^{n} X_{i} \leq n z\right\} & \text { for } \gamma>n z
\end{array} \quad \operatorname{Pr}\{\widehat{\boldsymbol{p}} \geq z\}= \begin{cases}\operatorname{Pr}\left\{\sum_{i=1}^{\lfloor\gamma / z\rfloor} X_{i} \geq \gamma\right\} & \text { for } \gamma \leq n z, \\
\operatorname{Pr}\left\{\sum_{i=1}^{n} X_{i} \geq n z\right\} & \text { for } \gamma>n z .\end{cases}\right.
$$

By applying Lemma 25, we can show the following lemma.

Lemma $26 \operatorname{Pr}\{\widehat{\boldsymbol{p}} \leq z \mid M\}$ is monotonically decreasing with respect to $M$. Similarly, $\operatorname{Pr}\{\widehat{\boldsymbol{p}} \geq z \mid$ $M\}$ is monotonically increasing with respect to $M$.

Now we shall introduce some new functions. Let $p_{0}<p_{1}<\cdots<p_{j}$ be all possible values of $\widehat{\boldsymbol{p}}$. Define random variable $R$ such that $\operatorname{Pr}\{R=r\}=\operatorname{Pr}\left\{\widehat{\boldsymbol{p}}=p_{r}\right\}$ for $r=0,1, \cdots, j$. Then, $\mathscr{U}(\widehat{\boldsymbol{p}})=\mathscr{U}\left(p_{R}\right)$. We denote $\mathscr{U}\left(p_{R}\right)$ as $\mathcal{U}(R)$. Clearly, $\mathcal{U}($.$) is a non-decreasing function defined$ on domain $\{0,1, \cdots, j\}$. By a linear interpolation, we can extend $\mathcal{U}($.$) as a continuous and$ non-decreasing function on $[0, j]$. Accordingly, we can define inverse function $\mathcal{U}^{-1}($.$) such that$ $\mathcal{U}^{-1}(\theta)=\max \{x \in[0, j]: \mathcal{U}(x)=\theta\}$ for $\mathscr{U}(0) \leq \theta \leq \mathscr{U}(j)$. Then, $\theta \geq \mathcal{U}(R) \Longleftrightarrow R \leq$ $\mathcal{U}^{-1}(\theta) \Longleftrightarrow R \leq g(\theta)$ where $g(\theta)=\left\lfloor\mathcal{U}^{-1}(\theta)\right\rfloor$.

Similarly, $\mathscr{L}(\widehat{\boldsymbol{p}})=\mathscr{L}\left(p_{R}\right)$. We denote $\mathscr{L}\left(p_{R}\right)$ as $\mathcal{L}(R)$. Clearly, $\mathcal{L}($.$) is a non-decreasing$ function defined on domain $\{0,1, \cdots, j\}$. By a linear interpolation, we can extend $\mathcal{L}($.$) as a$ continuous and non-decreasing function on $[0, j]$. Accordingly, we can define inverse function $\mathcal{L}^{-1}($.$) such that \mathcal{L}^{-1}(\theta)=\min \{x \in[0, j]: \mathcal{L}(x)=\theta\}$ for $\mathscr{L}(0) \leq \theta \leq \mathscr{L}(j)$. Then, $\theta \leq \mathcal{L}(R) \Longleftrightarrow$ $R \geq \mathcal{L}^{-1}(\theta) \Longleftrightarrow R \geq h(\theta)$ where $h(\theta)=\left\lceil\mathcal{L}^{-1}(\theta)\right\rceil$.

Lemma 27 Let $0 \leq r<j$. Then, $h(m)=r+1$ for $\mathcal{L}(r)<m \leq \mathcal{L}(r+1)$.

Proof. Clearly, $h(m)=r+1$ for $m=\mathcal{L}(r+1)$. It remains to evaluate $h(m)$ for $m$ satisfying $\mathcal{L}(r)<m<\mathcal{L}(r+1)$.

For $m>\mathcal{L}(r)$, we have $r<\mathcal{L}^{-1}(m)$, otherwise $r \geq \mathcal{L}^{-1}(m)$, implying $\mathcal{L}(r) \geq m$, since $\mathcal{L}($.$) is$ non-decreasing and $m \notin\{\mathcal{L}(r): 0 \leq r \leq j\}$. For $m<\mathcal{L}(r+1)$, we have $r+1>\mathcal{L}^{-1}(m)$, otherwise $r+1 \leq \mathcal{L}^{-1}(m)$, implying $\mathcal{L}(r+1) \leq m$, since $\mathcal{L}($.$) is non-decreasing and m \notin\{\mathcal{L}(r): 0 \leq r \leq j\}$. Therefore, we have $r<\mathcal{L}^{-1}(m)<r+1$ for $\mathcal{L}(r)<m<\mathcal{L}(r+1)$. Hence, $r<\left\lceil\mathcal{L}^{-1}(m)\right\rceil \leq r+1$, i.e., $r<h(m) \leq r+1$. Since $h(m)$ is an integer, we have $h(m)=r+1$ for $\mathcal{L}(r)<m<\mathcal{L}(r+1)$.

Lemma 28 Let $0 \leq r<j$. Then, $g(m)=r$ for $\mathcal{U}(r) \leq m<\mathcal{U}(r+1)$. 
Proof. Clearly, $g(m)=r$ for $m=\mathcal{U}(r)$. It remains to evaluate $g(m)$ for $m$ satisfying $\mathcal{U}(r)<$ $m<\mathcal{U}(r+1)$.

For $m>\mathcal{U}(r)$, we have $r<\mathcal{U}^{-1}(m)$, otherwise $r \geq \mathcal{U}^{-1}(m)$, implying $\mathcal{U}(r) \geq m$, since $\mathcal{U}($.$) is$ non-decreasing and $m \notin\{\mathcal{U}(r): 0 \leq r \leq j\}$. For $m<\mathcal{U}(r+1)$, we have $r+1>\mathcal{U}^{-1}(m)$, otherwise $r+1 \leq \mathcal{U}^{-1}(m)$, implying $\mathcal{U}(r+1) \leq m$, since $\mathcal{U}($.$) is non-decreasing and m \notin\{\mathcal{U}(r): 0 \leq r \leq j\}$. Therefore, for $\mathcal{U}(r)<m<\mathcal{U}(r+1)$, we have $r<\mathcal{U}^{-1}(m)<r+1$. Hence, $r \leq\left\lfloor\mathcal{U}^{-1}(m)\right\rfloor<r+1$, i.e., $r \leq g(m)<r+1$. Since $g(m)$ is an integer, we have $g(m)=r$ for $\mathcal{U}(r)<m<\mathcal{U}(r+1)$.

Noting that $\operatorname{Pr}\{M \geq \mathscr{U}(\widehat{\boldsymbol{p}}) \mid M\}=\operatorname{Pr}\{M \geq \mathcal{U}(R) \mid M\}$, we have $\operatorname{Pr}\{M \geq \mathscr{U}(\widehat{\boldsymbol{p}}) \mid M\}=$ $\operatorname{Pr}\{R \leq g(M) \mid M\}$. Let $0 \leq r<j$. By Lemma 28, we have that $g(m)=r$ for $\mathcal{U}(r) \leq m<$ $\mathcal{U}(r+1)$. Observing that $\operatorname{Pr}\{M \geq \mathscr{U}(\widehat{\boldsymbol{p}}) \mid M\}=0$ for $0 \leq M<\mathscr{U}(0)$ and that $\operatorname{Pr}\{M \geq \mathscr{U}(\widehat{\boldsymbol{p}}) \mid$ $M\}=1$ for $\mathscr{U}(j) \leq M \leq N$, we have that the maximum of $\operatorname{Pr}\{M \geq \mathscr{U}(\widehat{\boldsymbol{p}}) \mid M\}$ with respect to $M \in[a, b]$ is achieved on $\bigcup_{r=0}^{j-1}\{m \in[a, b]: \mathcal{U}(r) \leq m \leq \mathcal{U}(r+1)\} \cup\{a, b\}$. Now consider the range $\{m \in[a, b]: \mathcal{U}(r) \leq m \leq \mathcal{U}(r+1)\}$ of $M$. We only consider the non-trivial situation that $\mathcal{U}(r)<\mathcal{U}(r+1)$. For $\mathcal{U}(r) \leq M<\mathcal{U}(r+1)$, we have

$$
\operatorname{Pr}\{M \geq \mathscr{U}(\widehat{\boldsymbol{p}}) \mid M\}=\operatorname{Pr}\{R \leq g(M) \mid M\}=\operatorname{Pr}\{R \leq r \mid M\}=\operatorname{Pr}\left\{\widehat{\boldsymbol{p}} \leq p_{r} \mid M\right\}
$$

which is non-increasing for this range of $M$ as can be seen from Lemma 26. By virtue of such monotonicity, we can characterize the maximizer of $\operatorname{Pr}\{M \geq \mathscr{U}(\widehat{\boldsymbol{p}}) \mid M\}$ with respect to $M$ on the set $\{m \in[a, b]: \mathcal{U}(r) \leq m \leq \mathcal{U}(r+1)\}$ as follows.

Case (i): $b<\mathcal{U}(r)$ or $a>\mathcal{U}(r+1)$. This is trivial.

Case (ii): $a<\mathcal{U}(r) \leq b \leq \mathcal{U}(r+1)$. The maximizer must be among $\{\mathcal{U}(r), b\}$.

Case (iii): $\mathcal{U}(r) \leq a \leq b \leq \mathcal{U}(r+1)$. The maximizer must be among $\{a, b\}$.

Case (iv): $\mathcal{U}(r) \leq a \leq \mathcal{U}(r+1)<b$. The maximizer must be among $\{a, \mathcal{U}(r+1)\}$.

Case $(\mathrm{v}): a<\mathcal{U}(r) \leq \mathcal{U}(r+1)<b$. The maximizer must be among $\{\mathcal{U}(r), \mathcal{U}(r+1)\}$.

In summary, the maximizer must be among $\{\mathcal{U}(r), \mathcal{U}(r+1), a, b\} \cap[a, b]$. It follows that the statement on $\operatorname{Pr}\{M \geq \mathscr{U}(\widehat{\boldsymbol{p}}) \mid M\}$ is established.

Next, we consider $\operatorname{Pr}\{M \leq \mathscr{L}(\widehat{\boldsymbol{p}}) \mid M\}$. Noting that $\operatorname{Pr}\{M \leq \mathscr{L}(\widehat{\boldsymbol{p}}) \mid M\}=\operatorname{Pr}\{M \leq \mathcal{L}(R) \mid$ $M\}$, we have $\operatorname{Pr}\{M \leq \mathscr{L}(\widehat{\boldsymbol{p}}) \mid M\}=\operatorname{Pr}\{R \geq h(M) \mid M\}$. Let $0 \leq r<j$. By Lemma 27, we have that $h(m)=r+1$ for $\mathcal{L}(r)<m \leq \mathcal{L}(r+1)$. Observing that $\operatorname{Pr}\{M \leq \mathscr{L}(\widehat{\boldsymbol{p}}) \mid M\}=1$ for $0 \leq M \leq \mathscr{L}(0)$ and that $\operatorname{Pr}\{M \leq \mathscr{L}(\widehat{\boldsymbol{p}}) \mid M\}=0$ for $\mathscr{L}(j)<M \leq N$, we have that the maximum of $\operatorname{Pr}\{M \leq \mathscr{L}(\widehat{\boldsymbol{p}}) \mid M\}$ with respect to $M \in[a, b]$ is achieved on $\bigcup_{r=0}^{j-1}\{m \in[a, b]$ : $\mathcal{L}(r) \leq m \leq \mathcal{L}(r+1)\} \cup\{a, b\}$. Now consider the range $\{m \in[a, b]: \mathcal{L}(r) \leq m \leq \mathcal{L}(r+1)\}$ of $M$. We only consider the non-trivial situation that $\mathcal{L}(r)<\mathcal{L}(r+1)$. For $\mathcal{L}(r)<M \leq \mathcal{L}(r+1)$, we have

$$
\operatorname{Pr}\{M \leq \mathscr{L}(\widehat{\boldsymbol{p}}) \mid M\}=\operatorname{Pr}\{R \geq h(M) \mid M\}=\operatorname{Pr}\{R \geq r+1 \mid M\}=\operatorname{Pr}\left\{\widehat{\boldsymbol{p}} \geq p_{r+1} \mid M\right\},
$$


which is non-decreasing for this range of $M$ as can be seen from Lemma 26, By virtue of such monotonicity, we can characterize the maximizer of $\operatorname{Pr}\{M \leq \mathscr{L}(\widehat{\boldsymbol{p}}) \mid M\}$ with respect to $M$ on the set $\{m \in[a, b]: \mathcal{L}(r) \leq m \leq \mathcal{L}(r+1)\}$ as follows.

Case (i): $b<\mathcal{L}(r)$ or $a>\mathcal{L}(r+1)$. This is trivial.

Case (ii): $a<\mathcal{L}(r) \leq b \leq \mathcal{L}(r+1)$. The maximizer must be among $\{\mathcal{L}(r), b\}$.

Case (iii): $\mathcal{L}(r) \leq a \leq b \leq \mathcal{L}(r+1)$. The maximizer must be among $\{a, b\}$.

Case (iv): $\mathcal{L}(r) \leq a \leq \mathcal{L}(r+1)<b$. The maximizer must be among $\{a, \mathcal{L}(r+1)\}$.

Case $(\mathrm{v}): a<\mathcal{L}(r) \leq \mathcal{L}(r+1)<b$. The maximizer must be among $\{\mathcal{L}(r), \mathcal{L}(r+1)\}$.

In summary, the maximizer must be among $\{\mathcal{L}(r), \mathcal{L}(r+1), a, b\} \cap[a, b]$. It follows that the statement on $\operatorname{Pr}\{M \leq \mathscr{L}(\widehat{\boldsymbol{p}}) \mid M\}$ is established.

This concludes the proof of Theorem 8 .

\section{H Proof of Theorem 10}

The theorem can be established by showing the following lemmas.

Lemma $29 \operatorname{Pr}\left\{M>M_{u}\right\} \leq \frac{\delta}{2}$.

Proof. Since $X_{i}$ must be either 0 or 1 and $\gamma$ is an integer, we have $\operatorname{Pr}\left\{\sum_{i=1}^{[\gamma / z\rceil-1} X_{i}<\gamma\right\} \leq$ $\operatorname{Pr}\left\{\sum_{i=1}^{\lceil\gamma / z\rceil} X_{i} \leq \gamma\right\}$. Hence, by Lemma 25,

$$
\operatorname{Pr}\{\widehat{\boldsymbol{p}} \leq z\} \leq \begin{cases}\operatorname{Pr}\left\{\sum_{i=1}^{\lceil\gamma / z\rceil} X_{i} \leq \gamma\right\} & \text { for } \gamma \leq n z \\ \operatorname{Pr}\left\{\sum_{i=1}^{n} X_{i} \leq n z\right\} & \text { for } \gamma>n z\end{cases}
$$

and $\operatorname{Pr}\{\widehat{\boldsymbol{p}} \leq z\} \leq \mathscr{G}(z, p)$, where

$$
\mathscr{G}(z, p)= \begin{cases}\sum_{i=0}^{\gamma}\left(\begin{array}{c}
M \\
i
\end{array}\right)\left(\begin{array}{c}
N-M \\
\lceil\gamma / z\rceil-i
\end{array}\right) /\left(\begin{array}{c}
N \\
\lceil\gamma / z\rceil
\end{array}\right) & \text { for } \frac{\gamma}{n} \leq z \leq 1, \\
\sum_{i=0}^{\lfloor n z\rfloor}\left(\begin{array}{c}
M \\
i
\end{array}\right)\left(\begin{array}{c}
N-M \\
n-i
\end{array}\right) /\left(\begin{array}{c}
N \\
n
\end{array}\right) & \text { for } 0 \leq z<\frac{\gamma}{n}\end{cases}
$$

with $p=\frac{M}{N}$. Let $z^{*} \in[0,1]$ be the largest number such that $\operatorname{Pr}\left\{\widehat{\boldsymbol{p}}<z^{*}\right\} \leq \frac{\delta}{2}$. Since $\widehat{\boldsymbol{p}}$ is a discrete random variable bounded in $[0,1]$, it must be true that $\operatorname{Pr}\left\{\widehat{\boldsymbol{p}} \leq z^{*}\right\}>\frac{\delta}{2}$. Observing that $\mathscr{G}(z, p)$ is monotonically decreasing with respect to $p \in\left\{\frac{i}{N}: i=0,1, \cdots, N\right\}$, we have

$$
\{p \geq \overline{\boldsymbol{p}}\} \subseteq\left\{\mathscr{G}(\widehat{\boldsymbol{p}}, p) \leq \mathscr{G}(\widehat{\boldsymbol{p}}, \overline{\boldsymbol{p}}) \leq \frac{\delta}{2}\right\} \subseteq\left\{\mathscr{G}(\widehat{\boldsymbol{p}}, p) \leq \frac{\delta}{2}\right\}
$$

where $\overline{\boldsymbol{p}}=\frac{\boldsymbol{M}_{u}+1}{N}$. Noting that $\frac{\delta}{2}<\operatorname{Pr}\left\{\widehat{\boldsymbol{p}} \leq z^{*}\right\} \leq \mathscr{G}\left(z^{*}, p\right)$ and that $\mathscr{G}(z, p)$ is non-decreasing with respect to $z \in(0,1)$, we have $\{p \geq \overline{\boldsymbol{p}}\} \subseteq\left\{\mathscr{G}(\widehat{\boldsymbol{p}}, p) \leq \frac{\delta}{2}\right\} \subseteq\left\{\mathscr{G}(\widehat{\boldsymbol{p}}, p)<\mathscr{G}\left(z^{*}, p\right)\right\} \subseteq\left\{\widehat{\boldsymbol{p}}<z^{*}\right\}$. It follows that $\operatorname{Pr}\{p \geq \overline{\boldsymbol{p}}\} \leq \operatorname{Pr}\left\{\widehat{\boldsymbol{p}}<z^{*}\right\} \leq \frac{\delta}{2}$, which implies that $\operatorname{Pr}\left\{M>\boldsymbol{M}_{u}\right\} \leq \frac{\delta}{2}$.

Lemma $30 \operatorname{Pr}\left\{M<M_{l}\right\} \leq \frac{\delta}{2}$. 
Proof. By Lemma 25, we have $\operatorname{Pr}\{\widehat{\boldsymbol{p}} \geq z\}=\mathscr{H}(z, p)$, where

$$
\mathscr{H}(z, p)= \begin{cases}\sum_{i=\gamma}^{\lfloor\gamma / z\rfloor}\left(\begin{array}{c}
M \\
i
\end{array}\right)\left(\begin{array}{c}
N-M \\
\lfloor\gamma / z\rfloor-i
\end{array}\right) /\left(\begin{array}{c}
N \\
\lfloor\gamma / z\rfloor
\end{array}\right) & \text { for } \frac{\gamma}{n} \leq z \leq 1 \\
\sum_{i=\lceil n z\rceil}^{n}\left(\begin{array}{c}
M \\
i
\end{array}\right)\left(\begin{array}{c}
N-M \\
n-i
\end{array}\right) /\left(\begin{array}{c}
N \\
n
\end{array}\right) & \text { for } 0 \leq z<\frac{\gamma}{n}\end{cases}
$$

with $p=\frac{M}{N}$. Let $z^{*} \in[0,1]$ be the smallest number such that $\operatorname{Pr}\left\{\widehat{\boldsymbol{p}}>z^{*}\right\} \leq \frac{\delta}{2}$. Since $\widehat{\boldsymbol{p}}$ is a discrete random variable bounded in $[0,1]$, it must be true that $\operatorname{Pr}\left\{\widehat{\boldsymbol{p}} \geq z^{*}\right\}>\frac{\delta}{2}$. Observing that $\mathscr{H}(z, p)$ is monotonically increasing with respect to $p \in\left\{\frac{i}{N}: i=0,1, \cdots, N\right\}$, we have

$$
\{p \leq \underline{\boldsymbol{p}}\} \subseteq\left\{\mathscr{H}(\widehat{\boldsymbol{p}}, p) \leq \mathscr{H}(\widehat{\boldsymbol{p}}, \underline{\boldsymbol{p}}) \leq \frac{\delta}{2}\right\} \subseteq\left\{\mathscr{H}(\widehat{\boldsymbol{p}}, p) \leq \frac{\delta}{2}\right\}
$$

where $\underline{\boldsymbol{p}}=\frac{\boldsymbol{M}_{l}-1}{N}$. Noting that $\frac{\delta}{2}<\operatorname{Pr}\left\{\widehat{\boldsymbol{p}} \geq z^{*}\right\}=\mathscr{H}\left(z^{*}, p\right)$ and that $\mathscr{H}(z, p)$ is non-increasing with respect to $z \in(0,1)$, we have $\{p \leq \underline{\boldsymbol{p}}\} \subseteq\left\{\mathscr{H}(\widehat{\boldsymbol{p}}, p) \leq \frac{\delta}{2}\right\} \subseteq\left\{\mathscr{H}(\widehat{\boldsymbol{p}}, p)<\mathscr{H}\left(z^{*}, p\right)\right\} \subseteq\{\widehat{\boldsymbol{p}}>$ $\left.z^{*}\right\}$. It follows that $\operatorname{Pr}\{p \leq \underline{\boldsymbol{p}}\} \leq \operatorname{Pr}\left\{\widehat{\boldsymbol{p}}>z^{*}\right\} \leq \frac{\delta}{2}$, which implies $\operatorname{Pr}\left\{M<\boldsymbol{M}_{l}\right\} \leq \frac{\delta}{2}$.

\section{Proof of Theorem 11}

In the case of $M<\gamma$, we have $\mathbf{n}=n$ and $\frac{\gamma}{p}>\frac{\gamma N}{M}>N$, from which the theorem immediately follows. It remains to show the theorem for the case of $M \geq \gamma$. Notice that

$$
\begin{aligned}
\mathbb{E}[\mathbf{n}] & =n \operatorname{Pr}\left\{\sum_{i=1}^{n} X_{i}<\gamma\right\}+\sum_{m=1}^{n} m \operatorname{Pr}\left\{\sum_{i=1}^{m-1} X_{i}<\gamma, \sum_{i=1}^{m} X_{i} \geq \gamma\right\} \\
& <n \operatorname{Pr}\left\{\sum_{i=1}^{n} X_{i}<\gamma\right\}+\sum_{m=1}^{n} n \operatorname{Pr}\left\{\sum_{i=1}^{m-1} X_{i}<\gamma, \sum_{i=1}^{m} X_{i} \geq \gamma\right\}=n .
\end{aligned}
$$

Since $M=\sum_{i=1}^{N} X_{i} \geq \gamma$ and $X_{i}$ is non-negative, we have

$$
\bigcup_{m=n+1}^{N}\left\{\sum_{i=1}^{m-1} X_{i}<\gamma, \sum_{i=1}^{m} X_{i} \geq \gamma\right\}=\left\{\sum_{i=1}^{n} X_{i}<\gamma\right\} .
$$

Hence,

$$
\begin{aligned}
\mathbb{E}[\mathbf{n}] & =\sum_{m=n+1}^{N} n \operatorname{Pr}\left\{\sum_{i=1}^{m-1} X_{i}<\gamma, \sum_{i=1}^{m} X_{i} \geq \gamma\right\}+\sum_{m=1}^{n} m \operatorname{Pr}\left\{\sum_{i=1}^{m-1} X_{i}<\gamma, \sum_{i=1}^{m} X_{i} \geq \gamma\right\} \\
& <\sum_{m=1}^{N} m \operatorname{Pr}\left\{\sum_{i=1}^{m-1} X_{i}<\gamma, \sum_{i=1}^{m} X_{i} \geq \gamma\right\}=\mathbb{E}[\mathbf{m}],
\end{aligned}
$$

where $\mathbf{m}$ is the sample number of the classical inverse sampling scheme with the following stopping rule: Sampling without replacement is continued until $\gamma$ units possessing the attribute have been observed. By the definition of the classical inverse sampling, we have $\sum_{i=1}^{\mathbf{m}} X_{i}=\gamma$. Noting that $X_{1}, X_{2}, \cdots, X_{N}$ are identical but dependent Bernoulli random variables with common mean $p$ and 
that $\{\mathbf{n} \geq k\}$ depends only on $X_{1}, \cdots, X_{k-1}$ for $1 \leq k \leq N$, we can conclude that Wald's equation still applies. Hence, $\mathbb{E}\left[\sum_{i=1}^{\mathbf{m}} X_{i}\right]=\mathbb{E}[\mathbf{m}] \mathbb{E}\left[X_{i}\right]=\gamma$, which implies that $\mathbb{E}[\mathbf{m}]=\frac{\gamma}{\mathbb{E}\left[X_{i}\right]}=\frac{\gamma}{p}$. Since $\mathbb{E}[\mathbf{n}]$ is less than both $n$ and $\mathbb{E}[\mathbf{m}]$ as shown above, we have $\mathbb{E}[\mathbf{n}]<\min \left\{n, \frac{\gamma}{p}\right\}$. This completes the proof of the theorem.

\section{J Proof of Theorem 12}

The theorem can be established by showing the following lemmas.

Lemma $31 \operatorname{Pr}\{\lambda \geq \bar{\lambda}\} \leq \frac{\delta}{2}$.

Proof. By Theorem 1, we have

$$
\operatorname{Pr}\{\widehat{\boldsymbol{\lambda}} \leq z\}= \begin{cases}\operatorname{Pr}\left\{\sum_{i=1}^{\lceil\gamma / z\rceil-1} X_{i}<\gamma\right\} & \text { for } \gamma \leq n z \\ \operatorname{Pr}\left\{\sum_{i=1}^{n} X_{i} \leq n z\right\} & \text { for } \gamma>n z\end{cases}
$$

and thus $\operatorname{Pr}\{\widehat{\boldsymbol{\lambda}} \leq z\}=\mathscr{G}(z, \lambda)$, where

$$
\mathscr{G}(z, \lambda)= \begin{cases}\sum_{i=0}^{\gamma-1} \frac{1}{i !}\left[\left(\left\lceil\frac{\gamma}{z}\right\rceil-1\right) \lambda\right]^{i} \exp \left(-\left(\left\lceil\frac{\gamma}{z}\right\rceil-1\right) \lambda\right) & \text { for } z \geq \frac{\gamma}{n}, \\ \sum_{i=0}^{\lfloor n z\rfloor} \frac{1}{i !}(n \lambda)^{i} \exp (-n \lambda) & \text { for } 0 \leq z<\frac{\gamma}{n} .\end{cases}
$$

Let $z^{*} \geq 0$ be the largest number such that $\operatorname{Pr}\left\{\widehat{\boldsymbol{\lambda}}<z^{*}\right\} \leq \frac{\delta}{2}$. Since $\widehat{\boldsymbol{\lambda}}$ is a non-negative discrete random variable, it must be true that $\operatorname{Pr}\left\{\widehat{\boldsymbol{\lambda}} \leq z^{*}\right\}>\frac{\delta}{2}$. Observing that $\mathscr{G}(z, \lambda)$ is monotonically decreasing with respect to $\lambda \in(0, \infty)$, we have

$$
\{\lambda \geq \overline{\boldsymbol{\lambda}}\} \subseteq\left\{\mathscr{G}(\widehat{\boldsymbol{\lambda}}, \lambda) \leq \mathscr{G}(\widehat{\boldsymbol{\lambda}}, \overline{\boldsymbol{\lambda}})=\frac{\delta}{2}\right\} \subseteq\left\{\mathscr{G}(\widehat{\boldsymbol{\lambda}}, \lambda) \leq \frac{\delta}{2}\right\} .
$$

Noting that $\frac{\delta}{2}<\operatorname{Pr}\left\{\widehat{\boldsymbol{\lambda}} \leq z^{*}\right\}=\mathscr{G}\left(z^{*}, \lambda\right)$ and that $\mathscr{G}(z, \lambda)$ is non-decreasing with respect to $z \in(0, \infty)$, we have $\{\lambda \geq \overline{\boldsymbol{\lambda}}\} \subseteq\left\{\mathscr{G}(\widehat{\boldsymbol{\lambda}}, \lambda) \leq \frac{\delta}{2}\right\} \subseteq\left\{\mathscr{G}(\widehat{\boldsymbol{\lambda}}, \lambda)<\mathscr{G}\left(z^{*}, \lambda\right)\right\} \subseteq\left\{\widehat{\boldsymbol{\lambda}}<z^{*}\right\}$. It follows that $\operatorname{Pr}\{\lambda \geq \overline{\boldsymbol{\lambda}}\} \leq \operatorname{Pr}\left\{\widehat{\boldsymbol{\lambda}}<z^{*}\right\} \leq \frac{\delta}{2}$.

Lemma $32 \operatorname{Pr}\{\lambda \leq \underline{\boldsymbol{\lambda}}\} \leq \frac{\delta}{2}$

Proof. By Theorem 1, we have

$$
\operatorname{Pr}\{\hat{\boldsymbol{\lambda}} \geq z\}= \begin{cases}\operatorname{Pr}\left\{\sum_{i=1}^{\lfloor\gamma / z\rfloor} X_{i} \geq \gamma\right\} & \text { for } \gamma \leq n z \\ \operatorname{Pr}\left\{\sum_{i=1}^{n} X_{i} \geq n z\right\} & \text { for } \gamma>n z\end{cases}
$$

and thus $\operatorname{Pr}\{\widehat{\boldsymbol{\lambda}} \geq z\}=\mathscr{H}(z, \lambda)$ where

$$
\mathscr{H}(z, \lambda)= \begin{cases}\sum_{i=\gamma}^{\infty} \frac{1}{i !}\left(\left\lfloor\frac{\gamma}{z}\right\rfloor \lambda\right)^{i} \exp \left(-\left\lfloor\frac{\gamma}{z}\right\rfloor \lambda\right) & \text { for } z \geq \frac{\gamma}{n} \\ \sum_{i=\lceil n z\rceil}^{\infty} \frac{1}{i !}(n \lambda)^{i} \exp (-n \lambda) & \text { for } 0 \leq z<\frac{\gamma}{n}\end{cases}
$$


Let $z^{*} \geq 0$ be the smallest number such that $\operatorname{Pr}\left\{\widehat{\boldsymbol{\lambda}}>z^{*}\right\} \leq \frac{\delta}{2}$. Since $\widehat{\boldsymbol{\lambda}}$ is a non-negative discrete random variable, it must be true that $\operatorname{Pr}\left\{\widehat{\boldsymbol{\lambda}} \geq z^{*}\right\}>\frac{\delta}{2}$. Observing that $\mathscr{H}(z, \lambda)$ is monotonically increasing with respect to $\lambda \in(0, \infty)$, we have

$$
\{\lambda \leq \underline{\boldsymbol{\lambda}}\}=\{\lambda \leq \underline{\boldsymbol{\lambda}}, \mathbf{k}>0\} \subseteq\left\{\mathscr{H}(\widehat{\boldsymbol{\lambda}}, \lambda) \leq \mathscr{H}(\widehat{\boldsymbol{\lambda}}, \underline{\boldsymbol{\lambda}})=\frac{\delta}{2}\right\} \subseteq\left\{\mathscr{H}(\widehat{\boldsymbol{\lambda}}, \lambda) \leq \frac{\delta}{2}\right\}
$$

Noting that $\frac{\delta}{2}<\operatorname{Pr}\left\{\widehat{\boldsymbol{\lambda}} \geq z^{*}\right\}=\mathscr{H}\left(z^{*}, \lambda\right)$ and that $\mathscr{H}(z, \lambda)$ is non-increasing with respect to $z \in(0, \infty)$, we have $\{\lambda \leq \underline{\boldsymbol{\lambda}}\} \subseteq\left\{\mathscr{H}(\widehat{\boldsymbol{\lambda}}, \lambda) \leq \frac{\delta}{2}\right\} \subseteq\left\{\mathscr{H}(\widehat{\boldsymbol{\lambda}}, \lambda)<\mathscr{H}\left(z^{*}, \lambda\right)\right\} \subseteq\left\{\widehat{\boldsymbol{\lambda}}>z^{*}\right\}$. It follows that $\operatorname{Pr}\{\lambda \leq \underline{\boldsymbol{\lambda}}\} \leq \operatorname{Pr}\left\{\widehat{\boldsymbol{\lambda}}>z^{*}\right\} \leq \frac{\delta}{2}$.

\section{K Proof of Theorem 13}

By the same method as that of Lemma 14, we have

Lemma $33 \operatorname{Pr}\left\{\widehat{\boldsymbol{\mu}} \geq\left(1+\varepsilon_{r}\right) \mu\right\}<\frac{\delta}{2}$ for any $\mu \in\left(p^{\star}, 1\right)$.

Lemma 34 Let $0<\varepsilon_{r}<1, z=\left(1-\varepsilon_{r}\right) \mu$ and $\varepsilon^{\prime}=1-\frac{\gamma\left(1-\varepsilon_{r}\right)}{\gamma+\varepsilon_{r}-1}$. Suppose $\gamma>\frac{1-\varepsilon_{r}}{\varepsilon_{r}}$. Then, $\mathscr{M}_{\mathrm{I}}\left(\frac{z \gamma}{\gamma-z}, \mu\right)<\mathscr{M}_{\mathrm{I}}\left(\left(1-\varepsilon^{\prime}\right) \mu, \mu\right)$ for any $\mu \in(0,1)$.

Proof. As a consequence of $\gamma>\frac{1-\varepsilon_{r}}{\varepsilon_{r}}$, we have $0<\frac{z \gamma}{\gamma-z}<\mu$ for any $\mu \in(0,1)$. Since $\mathscr{M}_{\mathrm{I}}(w, \mu)$ is monotonically increasing with respect to $w \in(0, \mu)$, it suffices to show that $\frac{z \gamma}{\gamma-z}<\left(1-\varepsilon^{\prime}\right) \mu$ for any $\mu \in(0,1)$. That is, to show $\frac{\left(1-\varepsilon_{r}\right) \mu \gamma}{\gamma-\left(1-\varepsilon_{r}\right) \mu}<\left(1-\varepsilon^{\prime}\right) \mu, \forall \mu \in(0,1)$, i.e., $\frac{\left(1-\varepsilon_{r}\right) \gamma}{\gamma-\left(1-\varepsilon_{r}\right) \mu}<1-\varepsilon^{\prime}, \forall \mu \in(0,1)$. This follows from the definition of $\varepsilon^{\prime}$.

Lemma $35 \operatorname{Pr}\left\{\widehat{\boldsymbol{\mu}} \leq\left(1-\varepsilon_{r}\right) \mu\right\}<\frac{\delta}{2}$ for any $\mu \in\left(p^{\star}, 1\right)$.

Proof. To prove the lemma, we shall consider the following two cases:

Case (i): $\left(1-\varepsilon_{r}\right) \mu \geq \frac{\gamma}{n}$;

Case (ii): $\left(1-\varepsilon_{r}\right) \mu<\frac{\gamma}{n}$.

For Case (i), applying Theorem 1 with $z=\left(1-\varepsilon_{r}\right) \mu \geq \frac{\gamma}{n}$, we have $\operatorname{Pr}\left\{\widehat{\boldsymbol{\mu}} \leq\left(1-\varepsilon_{r}\right) \mu\right\}=$ $\operatorname{Pr}\left\{\sum_{i=1}^{[\gamma / z\rceil-1} X_{i}<\gamma\right\}$. As a consequence of $\gamma>\frac{1-\varepsilon_{r}}{\varepsilon_{r}}$, we have $0<\frac{\gamma}{\lceil\gamma / z\rceil-1} \leq \frac{z \gamma}{\gamma-z}<\mu$ for any $\mu \in(0,1)$. Hence, applying Lemma 5, we have

$$
\begin{aligned}
\operatorname{Pr}\left\{\widehat{\boldsymbol{\mu}} \leq\left(1-\varepsilon_{r}\right) \mu\right\} & \leq \exp \left((\lceil\gamma / z\rceil-1) \mathscr{M}_{\mathrm{B}}\left(\frac{\gamma}{\lceil\gamma / z\rceil-1}, \mu\right)\right) \\
& =\exp \left(\gamma \mathscr{M}_{\mathrm{I}}\left(\frac{\gamma}{\lceil\gamma / z\rceil-1}, \mu\right)\right) \leq \exp \left(\gamma \mathscr{M}_{\mathrm{I}}\left(\frac{\gamma z}{\gamma-z}, \mu\right)\right) .
\end{aligned}
$$


By Lemmas 34 and 6 ,

$$
\begin{aligned}
\operatorname{Pr}\left\{\widehat{\boldsymbol{\mu}} \leq\left(1-\varepsilon_{r}\right) \mu\right\} & \leq \exp \left(\gamma \mathscr{M}_{\mathrm{I}}\left(\left(1-\varepsilon^{\prime}\right) \mu, \mu\right)\right) \leq \exp \left(\gamma \mathscr{M}_{\mathrm{I}}\left(\left(1-\varepsilon^{\prime}\right) p^{\star}, p^{\star}\right)\right) \\
& =\exp \left(\gamma \mathscr{M}_{\mathrm{I}}\left(\frac{\gamma\left(1-\varepsilon_{r}\right) p^{\star}}{\gamma+\varepsilon_{r}-1}, p^{\star}\right)\right)<\frac{\delta}{2} .
\end{aligned}
$$

For Case (ii), applying Theorem 1 with $z=\left(1-\varepsilon_{r}\right) \mu<\frac{\gamma}{n}$ and by a similar argument as that of Lemma 15, we have $\operatorname{Pr}\left\{\widehat{\boldsymbol{\mu}} \leq\left(1-\varepsilon_{r}\right) \mu\right\}<\frac{\delta}{2}$.

In summary, we have shown $\operatorname{Pr}\left\{\widehat{\boldsymbol{\mu}} \leq\left(1-\varepsilon_{r}\right) \mu\right\}<\frac{\delta}{2}$ for all cases. The lemma is thus proved.

By the same method as that of Lemma 16, we have

Lemma $36 \operatorname{Pr}\left\{\widehat{\boldsymbol{\mu}} \geq \mu+\varepsilon_{a}\right\}<\frac{\delta}{2}$ for any $\mu \in\left(0, p^{\star}\right]$.

Lemma 37 Let $z=\mu-\varepsilon$. Suppose $0<\frac{z \gamma}{\gamma-z}<\mu$. Then, $\mathscr{M}_{\mathrm{I}}\left(\frac{z \gamma}{\gamma-z}, \mu\right)$ is monotonically increasing with respect to $\mu \in\left(\varepsilon, \frac{1}{2}\right)$.

Proof. Note that $\frac{\partial \mathscr{M}_{\mathrm{I}}(w, \mu)}{\partial \mu}=\frac{1}{\mu}-\frac{1}{w^{2}} \ln \left(\frac{1-\mu}{1-w}\right) \frac{\partial w}{\partial \mu}-\left(\frac{1}{w}-1\right) \frac{1}{1-\mu}$, where $w=\frac{z \gamma}{\gamma-z}=-\gamma+\frac{\gamma^{2}}{\gamma-z}$ and $\frac{\partial w}{\partial \mu}=\frac{\gamma^{2}}{(\gamma-z)^{2}}=\frac{w^{2}}{z^{2}}$. Hence,

$$
\begin{aligned}
\frac{\partial \mathscr{M}_{\mathrm{I}}(w, \mu)}{\partial \mu} & =\frac{1}{\mu}+\frac{1}{z^{2}} \ln \left(\frac{1-w}{1-\mu}\right)-\left(\frac{1}{w}-1\right) \frac{1}{1-\mu}>\frac{1}{\mu}+\frac{1}{z^{2}}\left(\frac{\mu-w}{1-w}\right)-\left(\frac{1}{w}-1\right) \frac{1}{1-\mu} \\
& =\frac{1}{z^{2}}\left(\frac{\mu-w}{1-w}\right)-\frac{\mu-w}{\mu(1-\mu) w}>0
\end{aligned}
$$

if $z^{2}(1-w)<\mu(1-\mu) w$, i.e., $z^{2}\left(\frac{1}{w}-1\right)<\mu(1-\mu) \Longleftrightarrow z^{2}\left(\frac{1}{z}-\frac{1}{\gamma}-1\right)<\mu(1-\mu) \Longleftrightarrow z(1-z)-\frac{z^{2}}{\gamma}<$ $\mu(1-\mu)$ since $\frac{1}{w}=\frac{1}{z}-\frac{1}{\gamma}$.

Lemma $38 \operatorname{Pr}\left\{\widehat{\boldsymbol{\mu}} \leq \mu-\varepsilon_{a}\right\}<\frac{\delta}{2}$ for any $\mu \in\left(0, p^{\star}\right]$.

Proof. To prove the lemma, we shall consider the following three cases:

Case (i): $\mu<\varepsilon_{a}$;

Case (ii): $\mu-\varepsilon_{a} \geq \frac{\gamma}{n}$;

Case (iii): $0 \leq \mu-\varepsilon_{a}<\frac{\gamma}{n}$.

For Case (i), it is evident that $\operatorname{Pr}\left\{\widehat{\boldsymbol{\mu}} \leq \mu-\varepsilon_{a}\right\}=0<\frac{\delta}{2}$.

For Case (ii), applying Theorem 1 with $z=\mu-\varepsilon_{a} \geq \frac{\gamma}{n}$, we have $\operatorname{Pr}\left\{\widehat{\boldsymbol{\mu}} \leq \mu-\varepsilon_{a}\right\}=$ $\operatorname{Pr}\left\{\sum_{i=1}^{[\gamma / z\rceil-1} X_{i}<\gamma\right\}$. From the definition of $z$ and the assumption that $\gamma>\frac{1-\varepsilon_{r}}{\varepsilon_{r}}$, we see that $0<\frac{\gamma}{\lceil\gamma / z\rceil-1} \leq \frac{z \gamma}{\gamma-z}<\mu$ for any $\mu \in\left(0, p^{\star}\right]$. Hence, it follows from Lemma 5 that

$$
\begin{aligned}
\operatorname{Pr}\left\{\widehat{\boldsymbol{\mu}} \leq \mu-\varepsilon_{a}\right\} & \leq \exp \left((\lceil\gamma / z\rceil-1) \mathscr{M}_{\mathrm{B}}\left(\frac{\gamma}{\lceil\gamma / z\rceil-1}, \mu\right)\right) \\
& =\exp \left(\gamma \mathscr{M}_{\mathrm{I}}\left(\frac{\gamma}{\lceil\gamma / z\rceil-1}, \mu\right)\right) \leq \exp \left(\gamma \mathscr{M}_{\mathrm{I}}\left(\frac{\gamma z}{\gamma-z}, \mu\right)\right) .
\end{aligned}
$$


Invoking Lemma 37, we have

$$
\begin{aligned}
\operatorname{Pr}\left\{\widehat{\boldsymbol{\mu}} \leq \mu-\varepsilon_{a}\right\} & \leq \exp \left(\gamma \mathscr{M}_{\mathrm{I}}\left(\frac{\gamma\left(p^{\star}-\varepsilon_{a}\right)}{\gamma-\left(p^{\star}-\varepsilon_{a}\right)}, p^{\star}\right)\right)=\exp \left(\gamma \mathscr{M}_{\mathrm{I}}\left(\frac{\gamma\left(1-\varepsilon_{r}\right) p^{\star}}{\gamma-\left(1-\varepsilon_{r}\right) p^{\star}}, p^{\star}\right)\right) \\
& <\exp \left(\gamma \mathscr{M}_{\mathrm{I}}\left(\left(1-\varepsilon^{\prime}\right) p^{\star}, p^{\star}\right)\right)=\exp \left(\gamma \mathscr{M}_{\mathrm{I}}\left(\frac{\gamma\left(1-\varepsilon_{r}\right) p^{\star}}{\gamma+\varepsilon_{r}-1}, p^{\star}\right)\right)<\frac{\delta}{2}
\end{aligned}
$$

where $\varepsilon^{\prime}$ is defined in Lemma 34 .

For Case (iii), applying Theorem 1 with $z=\mu-\varepsilon_{a}<\frac{\gamma}{n}$ and by an argument as that of Lemma 17. we have $\operatorname{Pr}\left\{\widehat{\boldsymbol{\mu}} \leq \mu-\varepsilon_{a}\right\}<\frac{\delta}{2}$. In summary, we have shown $\operatorname{Pr}\left\{\widehat{\boldsymbol{\mu}} \leq \mu-\varepsilon_{a}\right\}<\frac{\delta}{2}$ for all cases. The lemma is thus proved.

Finally, the proof of Theorem 13 can be accomplished by a similar argument as that of Theorem 3.

\section{Proof of Theorem 14}

The theorem can be established by showing the following lemmas.

Lemma $39 \operatorname{Pr}\{\mu \geq \overline{\boldsymbol{\mu}}\} \leq \frac{\delta}{2}$.

Proof. For $\mu>z \geq \frac{\gamma}{n}$, by Theorem 1 and Lemma 5, we have

$$
\begin{aligned}
\operatorname{Pr}\{\widehat{\boldsymbol{\mu}} \leq z\} & =\operatorname{Pr}\left\{\sum_{i=1}^{\lceil\gamma / z\rceil-1} X_{i}<\gamma\right\} \leq \exp \left((\lceil\gamma / z\rceil-1) \mathscr{M}_{\mathrm{B}}\left(\frac{\gamma}{\lceil\gamma / z\rceil-1}, \mu\right)\right) \\
& =\exp \left(\gamma \mathscr{M}_{\mathrm{I}}\left(\frac{\gamma}{\lceil\gamma / z\rceil-1}, \mu\right)\right) \leq \exp \left(\gamma \mathscr{M}_{\mathrm{I}}\left(\frac{z \gamma}{\gamma-z}, \mu\right)\right)
\end{aligned}
$$

where the last inequality is due to $\frac{\gamma}{|\gamma / z|-1} \leq \frac{z \gamma}{\gamma-z}$ and the fact that $\mathscr{M}_{\mathrm{I}}(z, \mu)$ is monotonically increasing with respect to $z \in(0, \mu)$. For $\mu>z$ and $0 \leq z<\frac{\gamma}{n}$, by Theorem 1 and Lemma 5 , we have $\operatorname{Pr}\{\widehat{\boldsymbol{\mu}} \leq z\}=\operatorname{Pr}\left\{\frac{\sum_{i=1}^{n} X_{i}}{n} \leq z\right\} \leq \exp \left(n \mathscr{M}_{\mathrm{B}}(z, \mu)\right)$. Therefore, $\operatorname{Pr}\{\widehat{\boldsymbol{\mu}} \leq z\} \leq \mathscr{G}(z, \mu)$, where

$$
\mathscr{G}(z, \mu)= \begin{cases}\exp \left(\gamma \mathscr{M}_{\mathrm{I}}\left(\frac{z \gamma}{\gamma-z}, \mu\right)\right) & \text { for } \frac{\gamma}{n} \leq z<\mu, \\ \exp \left(n \mathscr{M}_{\mathrm{B}}(z, \mu)\right) & \text { for } 0 \leq z<\frac{\gamma}{n}, z<\mu .\end{cases}
$$

Let $z^{*} \in[0,1]$ be the largest number such that $\operatorname{Pr}\left\{\widehat{\boldsymbol{\mu}}<z^{*}\right\} \leq \frac{\delta}{2}$. Then, it must be true that either $\operatorname{Pr}\left\{\widehat{\boldsymbol{\mu}} \leq z^{*}\right\}>\frac{\delta}{2}$ or $\operatorname{Pr}\left\{\widehat{\boldsymbol{\mu}} \leq z^{*}\right\}=\frac{\delta}{2}$. Observing that $\mathscr{G}(z, \mu)$ is monotonically decreasing with respect to $\mu \in(z, 1)$, we have

$$
\{\mu \geq \overline{\boldsymbol{\mu}}\}=\{\mu \geq \overline{\boldsymbol{\mu}} \geq \widehat{\boldsymbol{\mu}}, \mathbf{k}<\mathbf{n}\} \subseteq\left\{\mathscr{G}(\widehat{\boldsymbol{\mu}}, \mu) \leq \mathscr{G}(\widehat{\boldsymbol{\mu}}, \overline{\boldsymbol{\mu}})=\frac{\delta}{2}, \mu \geq \overline{\boldsymbol{\mu}} \geq \widehat{\boldsymbol{\mu}}\right\} \subseteq\left\{\mathscr{G}(\widehat{\boldsymbol{\mu}}, \mu) \leq \frac{\delta}{2}, \widehat{\boldsymbol{\mu}} \leq \mu\right\} .
$$

In the case of $\operatorname{Pr}\left\{\widehat{\boldsymbol{\mu}} \leq z^{*}\right\}>\frac{\delta}{2}$, we have $\frac{\delta}{2}<\operatorname{Pr}\left\{\widehat{\boldsymbol{\mu}} \leq z^{*}\right\} \leq \mathscr{G}\left(z^{*}, \mu\right)$. Since $\mathscr{G}(z, \mu)$ is increasing with respect to $z \in(0, \mu)$, we have $\{\mu \geq \overline{\boldsymbol{\mu}}\} \subseteq\left\{\mathscr{G}(\widehat{\boldsymbol{\mu}}, \mu) \leq \frac{\delta}{2}, \widehat{\boldsymbol{\mu}} \leq \mu\right\} \subseteq\left\{\mathscr{G}(\widehat{\boldsymbol{\mu}}, \mu)<\mathscr{G}\left(z^{*}, \mu\right), \widehat{\boldsymbol{\mu}} \leq\right.$ 
$\mu\} \subseteq\left\{\widehat{\boldsymbol{\mu}}<z^{*}\right\}$. It follows that $\operatorname{Pr}\{\mu \geq \overline{\boldsymbol{\mu}}\} \leq \operatorname{Pr}\left\{\widehat{\boldsymbol{\mu}}<z^{*}\right\} \leq \frac{\delta}{2}$. In the case of $\operatorname{Pr}\left\{\widehat{\boldsymbol{\mu}} \leq z^{*}\right\}=\frac{\delta}{2}$, we have $\frac{\delta}{2}=\operatorname{Pr}\left\{\widehat{\boldsymbol{\mu}} \leq z^{*}\right\} \leq \mathscr{G}\left(z^{*}, \mu\right)$. Since $\mathscr{G}(z, \mu)$ is increasing with respect to $z \in(0, \mu)$, we have $\{\mu \geq \overline{\boldsymbol{\mu}}\} \subseteq\left\{\mathscr{G}(\widehat{\boldsymbol{\mu}}, \mu) \leq \frac{\delta}{2}, \widehat{\boldsymbol{\mu}} \leq \mu\right\} \subseteq\left\{\mathscr{G}(\widehat{\boldsymbol{\mu}}, \mu) \leq \mathscr{G}\left(z^{*}, \mu\right), \widehat{\boldsymbol{\mu}} \leq \mu\right\} \subseteq\left\{\widehat{\boldsymbol{\mu}} \leq z^{*}\right\}$. It follows that $\operatorname{Pr}\{\mu \geq \overline{\boldsymbol{\mu}}\} \leq \operatorname{Pr}\left\{\widehat{\boldsymbol{\mu}} \leq z^{*}\right\}=\frac{\delta}{2}$.

Lemma $40 \operatorname{Pr}\{\mu \leq \underline{\boldsymbol{\mu}}\} \leq \frac{\delta}{2}$.

Proof. For $z>\mu$ and $1 \geq z \geq \frac{\gamma}{n}$, by Theorem 1 and Lemma 5, we have

$$
\begin{aligned}
\operatorname{Pr}\{\widehat{\boldsymbol{\mu}} \geq z\} & =\operatorname{Pr}\left\{\sum_{i=1}^{\lfloor\gamma / z\rfloor} X_{i} \geq \gamma\right\} \leq \exp \left(\lfloor\gamma / z\rfloor \mathscr{M}_{\mathrm{B}}\left(\frac{\gamma}{\lfloor\gamma / z\rfloor}, \mu\right)\right) \\
& =\exp \left(\gamma \mathscr{M}_{\mathrm{I}}\left(\frac{\gamma}{\lfloor\gamma / z\rfloor}, \mu\right)\right) \leq \exp \left(\gamma \mathscr{M}_{\mathrm{I}}(z, \mu)\right)
\end{aligned}
$$

where the last inequality is due to $\frac{\gamma}{[\gamma / z]} \geq z$ and the fact that $\mathscr{M}_{\mathrm{I}}(z, \mu)$ is monotonically decreasing with respect to $z \in(\mu, 1)$. For $\mu<z<\frac{\gamma}{n}$, by Theorem 1 and Lemma 5, we have $\operatorname{Pr}\{\widehat{\boldsymbol{\mu}} \geq z\}=$ $\operatorname{Pr}\left\{\frac{\sum_{i=1}^{n} X_{i}}{n} \geq z\right\} \leq \exp \left(n \mathscr{M}_{\mathrm{B}}(z, \mu)\right)$. Therefore, $\operatorname{Pr}\{\widehat{\boldsymbol{\mu}} \geq z\} \leq \mathscr{H}(z, \mu)$, where

$$
\mathscr{H}(z, \mu)= \begin{cases}\exp \left(\gamma \mathscr{M}_{\mathrm{I}}(z, \mu)\right) & \text { for } 1 \geq z \geq \frac{\gamma}{n}, z>\mu \\ \exp \left(n \mathscr{M}_{\mathrm{B}}(z, \mu)\right) & \text { for } \mu<z<\frac{\gamma}{n}\end{cases}
$$

Let $z^{*} \in[0,1]$ be the smallest number such that $\operatorname{Pr}\left\{\widehat{\boldsymbol{\mu}}>z^{*}\right\} \leq \frac{\delta}{2}$. Then, it must be true that either $\operatorname{Pr}\left\{\widehat{\boldsymbol{\mu}} \geq z^{*}\right\}>\frac{\delta}{2}$ or $\operatorname{Pr}\left\{\widehat{\boldsymbol{\mu}} \geq z^{*}\right\}=\frac{\delta}{2}$. Observing that $\mathscr{H}(z, \mu)$ is monotonically increasing with respect to $\mu \in(0, z)$, we have

$$
\{\mu \leq \underline{\boldsymbol{\mu}}\}=\{\mu \leq \underline{\boldsymbol{\mu}} \leq \widehat{\boldsymbol{\mu}}, \mathbf{k}>0\} \subseteq\left\{\mathscr{H}(\widehat{\boldsymbol{\mu}}, \mu) \leq \mathscr{H}(\widehat{\boldsymbol{\mu}}, \underline{\boldsymbol{\mu}})=\frac{\delta}{2}, \mu \leq \underline{\boldsymbol{\mu}} \leq \widehat{\boldsymbol{\mu}}\right\} \subseteq\left\{\mathscr{H}(\widehat{\boldsymbol{\mu}}, \mu) \leq \frac{\delta}{2}, \widehat{\boldsymbol{\mu}} \geq \mu\right\} .
$$

In the case of $\operatorname{Pr}\left\{\widehat{\boldsymbol{\mu}} \geq z^{*}\right\}>\frac{\delta}{2}$, we have $\frac{\delta}{2}<\operatorname{Pr}\left\{\widehat{\boldsymbol{\mu}} \geq z^{*}\right\} \leq \mathscr{H}\left(z^{*}, \mu\right)$. Since $\mathscr{H}(z, \mu)$ is decreasing with respect to $z \in(\mu, 1)$, we have $\{\mu \leq \underline{\boldsymbol{\mu}}\} \subseteq\left\{\mathscr{H}(\widehat{\boldsymbol{\mu}}, \mu) \leq \frac{\delta}{2}, \widehat{\boldsymbol{\mu}} \geq \mu\right\} \subseteq\{\mathscr{H}(\widehat{\boldsymbol{\mu}}, \mu)<$ $\left.\mathscr{H}\left(z^{*}, \mu\right), \widehat{\boldsymbol{\mu}} \geq \mu\right\} \subseteq\left\{\widehat{\boldsymbol{\mu}}>z^{*}\right\}$. It follows that $\operatorname{Pr}\{\mu \leq \underline{\boldsymbol{\mu}}\} \leq \operatorname{Pr}\left\{\widehat{\boldsymbol{\mu}}>z^{*}\right\} \leq \frac{\delta}{2}$. In the case of $\operatorname{Pr}\left\{\widehat{\boldsymbol{\mu}} \geq z^{*}\right\}=\frac{\delta}{2}$, we have $\frac{\delta}{2}=\operatorname{Pr}\left\{\widehat{\boldsymbol{\mu}} \geq z^{*}\right\} \leq \mathscr{H}\left(z^{*}, \mu\right)$. Since $\mathscr{H}(z, \mu)$ is decreasing with respect to $z \in(\mu, 1)$, we have $\{\mu \leq \underline{\boldsymbol{\mu}}\} \subseteq\left\{\mathscr{H}(\widehat{\boldsymbol{\mu}}, \mu) \leq \frac{\delta}{2}, \widehat{\boldsymbol{\mu}} \geq \mu\right\} \subseteq\left\{\mathscr{H}(\widehat{\boldsymbol{\mu}}, \mu) \leq \mathscr{H}\left(z^{*}, \mu\right), \widehat{\boldsymbol{\mu}} \geq \mu\right\} \subseteq$ $\left\{\widehat{\boldsymbol{\mu}} \geq z^{*}\right\}$. It follows that $\operatorname{Pr}\{\mu \leq \underline{\boldsymbol{\mu}}\} \leq \operatorname{Pr}\left\{\widehat{\boldsymbol{\mu}} \geq z^{*}\right\}=\frac{\delta}{2}$.

\section{References}

[1] X. Chen, "Inverse sampling for nonasymptotic sequential estimation of bounded variable means," arXiv:0711.2801, November 2007. 
[2] X. Chen, "Interval estimation of bounded variable means via inverse sampling," arXiv:0802.3539, February 2008.

[3] J. Cheng, "Sampling algorithms for estimating the mean of bounded variables," Comput. Statist., vol. 16, pp. 1-23, 2001.

[4] C. J. Clopper and E. S. Pearson, "The use of confidence or fiducial limits illustrated in the case of the binomial," Biometrika, vol. 26, pp. 404-413, 1934.

[5] P. Dagum, R. Karp, M. Luby and S. Ross, "An optimal algorithm for Monte Carlo estimation," SIAM J. Comput., vol. 29, pp. 1484-1496, 2000.

[6] F. Garwood, "Fiducial limits for the Poisson distribution," Biometrika, vol. 28, pp. 437-442, 1936.

[7] W. Hoeffding, "Probability inequalities for sums of bounded variables," J. Amer. Statist. Assoc., vol. 58, pp. 13-29, 1963.

[8] J. B. S., Haldane, "A labour-saving method of sampling," Nature, vol. 155, pp. 49-50, January $13,1945$.

[9] J. B. S., Haldane, "On a method of estimating frequencies," Biometrika, vol. 33, pp. 222-225, 1945.

[10] L. Mendo and J. M. Hernando, "Estimation of a probability with optimum guaranteed confidence in inverse binomial sampling," arXiv:0809.2402, September 2008. 